\title{
Alternative Approaches to Estimation and Inference in Large Multifactor Panels: Small Sample Results with an Application to Modelling of Asset Returns
}

G. Kapetanios and M. Hashem Pesaran

May 2005

CWPE 0520

Not to be quoted without permission 


\title{
Alternative Approaches to Estimation and Inference in Large Multifactor Panels: Small Sample Results with an Application to Modelling of Asset Returns*
}

\author{
G. Kapetanios \\ Queen Mary, University of London
}

\author{
M. Hashem Pesaran \\ Cambridge University and USC
}

December 14, 2004

\begin{abstract}
This paper considers alternative approaches to the analysis of large panel data models in the presence of error cross section dependence. A popular method for modelling such dependence uses a factor error structure. Such models raise new problems for estimation and inference. This paper compares two alternative methods for carrying out estimation and inference in panels with a multifactor error structure. One uses the correlated common effects estimator that proxies the unobserved factors by cross section averages of the observed variables as suggested by Pesaran (2004), and the other uses principal components following the work of Stock and Watson (2002) . The paper develops the principal component method and provides small sample evidence on the comparative properties of these estimators by means of extensive Monte Carlo experiments. An empirical application to company returns provides an illustration of the alternative estimation procedures.
\end{abstract}

Keywords: Cross Section Dependence, Large Panels, Principal Components, Common Correlated Effects, Return Equations.

JEL-Classification: C12, C13, C33.

${ }^{*}$ We are grateful to Til Schuermann and Bejorn Treutler for providing us with the company data used in the empirical section. We have also benefited from comments and discussions with Ron Smith and Takashi Yagamata. 
Panel data sets have been increasingly used in economics to analyze complex economic phenomena. One of their attractions is the ability to use an extended data set to obtain information about parameters of interest which are assumed to have common values across panel units. Most of the work carried out on panel data has usually assumed some form of cross sectional independence to derive the theoretical properties of various inferential procedures. However, such assumptions are often suspect and as a result recent advances in the literature have focused on estimation of panel data models subject to error cross sectional dependence.

A number of different approaches have been advanced for this purpose. In the case of spatial data sets where a natural immutable distance measure is available the dependence is often captured through "spatial lags" using techniques familiar from the time series literature. In economic applications, spatial techniques are often adapted using alternative measures of "economic distance". This approach is exemplified in work by Lee and Pesaran (1993) , Conley and Dupor (2003), Conley and Topa (2002) and Pesaran, Schuermann, and Weiner (2004), as well as the literature on spatial econometrics recently surveyed by Anselin (2001) . In the case of panel data models where the cross section dimension $(N)$ is small (typically $N<10$ ) and the time series dimension $(T)$ is large the standard approach is to treat the equations from the different cross section units as a system of seemingly unrelated regression equations (SURE) and then estimate the system by the Generalized Least Squares (GLS) techniques.

In the case of panels with large cross section dimension, SURE approach is not practical and has led a number of investigators to consider unobserved factor models, where the cross section error correlations are defined in terms of the factor loadings. Use of factor models is not new in economics and dates back to the pioneering work of Stone (1947) who applied the principal components (PC) analysis of Hotelling to US macroeconomic time series over the period 1922-1938 and was able to demonstrate that three factors (namely total income, its rate of change and a time trend) explained over 97 per cent of the total variations of all the 17 macro variables that he had considered. Until recently, subsequent applications of the PC approach to economic times series has been primarily in finance. See, for example, Chamberlain and Rothschild (1983), Conor and Korajzcyk (1986) and Conor and Korajzcyk (1988) . But more recently the unobserved factor models have gained popularity for forecasting with a large number of variables as advocated by Stock and Watson (2002) - The factor model is used very much in the spirit of the original work by Stone, in order to summarize the empirical content of a large number of macroeconomics variables by 
a small set of factors which, when estimated using principal components, is then used for further modelling and/or forecasting. A related literature on dynamic factor models has also been put forward by Forni and Reichlin (1998) and Forni, Hallin, Lippi, and Reichlin (2000) .

Recent uses of factor models in forecasting focuses on consistent estimation of unobserved factors and their loadings. Related theoretical advances by Bai and Ng (2002) and Bai (2003) are also concerned with estimation and selection of unobserved factors and do not consider the estimation and inference problems in standard panel data models where the objects of interest are slope coefficients of the conditioning variables (regressors). In such panels the unobserved factors are viewed as nuisance variables, introduced primarily to model the cross section dependencies of the error terms in a parsimonious manner relative to the SURE formulation.

Despite these differences knowledge of factor models could still be useful for the analysis of panel data models if it is believed that the errors might be cross sectionally correlated. Disregarding the possible factor structure of the errors in panel data models can lead to inconsistent parameter estimates and incorrect inference. Coakley, Fuertes, and Smith (2002) suggest a possible solution to the problem using the method of Stock and Watson (2002) . But, as Pesaran (2004) shows, the PC approach proposed by Coakley, Fuertes, and Smith (2002) can still yield inconsistent estimates. Pesaran (2004) suggests a new approach by noting that linear combinations of the unobserved factors can be well approximated by cross section averages of the dependent variable and the observed regressors. This leads to a new set of estimators, referred to as the Common Correlated Effects estimators, that can be computed by running standard panel regressions augmented with the cross section averages of the dependent and independent variables. The CCE procedure is applicable to panels with a single or multiple unobserved factors so long as the number of unobserved factors is fixed.

In this paper we consider an alternative two-stage estimation method where in the first stage principal components of all the economic variables in the panel data model are obtained as in Stock and Watson (2002), and in the second stage the model is estimated augmenting the observed regressors with the estimated PCs. Unlike the CCE method the implementation of the $\mathrm{PC}$ augmented procedure requires the determination of the number of factors to be included in the second stage. This can be done using the criteria advanced in Bai and $\mathrm{Ng}(2002)$.

The small sample properties of the CCE and the PC augmented estimators will be inves- 
tigated by means of Monte Carlo experiments, allowing for up to four factors and regressors. We find that augmenting the panel data model with cross sectional averages of the dependent and explanatory variable works well in the multiple factor case. This is line with the results of Monte Carlo experiments reported by Pesaran (2004) and Coakley, Fuertes, and Smith (2004). On the other hand the PC augmented method does not perform as well, and can lead to substantial size distortions. This could be partly due to the small sample errors in the number of factors selected by the Bai and $\mathrm{Ng}$ procedure. To shed light on such a possibility we also conducted a number of Monte Carlo experiments where the factors were taken as observed, but it is not known which of the factors should actually be included in the PC augmented procedure. Using alternative regressor selection procedures it is shown that even in this setting the PC augmented method could be subject to substantial bias in small samples. We also provide an empirical application to a large panel of company returns with a wide geographical coverage where we estimate asset return regressions that include observed as well as unobserved regressors. The standard asset return equations routinely estimated in the finance literature either allow for unobserved factors or observed factors, but not both. We extend this literature by including both types of regressors in the analysis.

The plan of the paper is as follows: Section 1 sets out the multi-factor residual model, its assumptions and the CCE estimators. Section 2 sets out the PC augmented estimators. Section 3 describes the Monte Carlo design and discusses the results. Section 4 presents the empirical application. Finally, Section 5 concludes.

\section{Panel Data Models with Observed and Unobserved Common Effects}

In this section we review the methodology introduced in Pesaran (2004) . Let $y_{i t}$ be the observation on the $i^{t h}$ cross section unit at time $t$ for $i=1,2, \ldots, N ; t=1,2, \ldots, T$, and suppose that it is generated according to the following linear heterogeneous panel data model

$$
y_{i t}=\boldsymbol{\alpha}_{i}^{\prime} \mathbf{d}_{t}+\boldsymbol{\beta}_{i}^{\prime} \mathbf{x}_{i t}+\boldsymbol{\gamma}_{i}^{\prime} \mathbf{f}_{t}+\varepsilon_{i t},
$$

where $\mathbf{d}_{t}$ is a $n \times 1$ vector of observed common effects (including deterministic components such as intercepts or seasonal dummies), $\mathbf{x}_{i t}$ is a $k \times 1$ vector of observed individual-specific regressors on the $i^{t h}$ cross section unit at time $t, \mathbf{f}_{t}$ is the $m \times 1$ vector of unobserved common effects, and $\varepsilon_{i t}$ are the individual-specific (idiosyncratic) errors assumed to be independently distributed of $\left(\mathbf{d}_{t}, \mathbf{x}_{i t}\right)$. The unobserved factors, $\mathbf{f}_{t}$, could be correlated with $\left(\mathbf{d}_{t}, \mathbf{x}_{i t}\right)$, and to allow for such a possibility the following specification for the individual specific regressors 
will be considered

$$
\mathbf{x}_{i t}=\mathbf{A}_{i}^{\prime} \mathbf{d}_{t}+\Gamma_{i}^{\prime} \mathbf{f}_{t}+\mathbf{v}_{i t}
$$

where $\mathbf{A}_{i}$ and $\boldsymbol{\Gamma}_{i}$ are $n \times k$ and $m \times k$, factor loading matrices with fixed components, $\mathbf{v}_{i t}$ are the specific components of $\mathbf{x}_{i t}$ distributed independently of the common effects and across $i$, but assumed to follow general covariance stationary processes. In this paper we assume that the common factors, $\mathbf{d}_{t}$ and $\mathbf{f}_{t}$, are covariance stationary, although the results obtained here can be readily extended to cases where one or more elements of the common factors could have unit roots and/or deterministic trends.

Combining (1) and (2) we now have

$$
\underset{(k+1) \times 1}{\mathbf{z}_{i t}}=\left(\begin{array}{c}
y_{i t} \\
\mathbf{x}_{i t}
\end{array}\right)=\underset{(k+1) \times n}{\mathbf{B}_{i}^{\prime}} \underset{n \times 1}{\mathbf{d}_{t}}+\underset{(k+1) \times m}{\mathbf{C}_{i}^{\prime}} \underset{m \times 1}{\mathbf{f}_{t}}+\underset{(k+1) \times 1}{\mathbf{u}_{i t}},
$$

where

$$
\begin{gathered}
\mathbf{u}_{i t}=\left(\begin{array}{c}
\varepsilon_{i t}+\boldsymbol{\beta}_{i}^{\prime} \mathbf{v}_{i t} \\
\mathbf{v}_{i t}
\end{array}\right), \\
\mathbf{B}_{i}=\left(\begin{array}{ll}
\boldsymbol{\alpha}_{i} & \mathbf{A}_{i}
\end{array}\right)\left(\begin{array}{cc}
1 & \mathbf{0} \\
\boldsymbol{\beta}_{i} & \mathbf{I}_{k}
\end{array}\right), \mathbf{C}_{i}=\left(\begin{array}{ll}
\boldsymbol{\gamma}_{i} & \boldsymbol{\Gamma}_{i}
\end{array}\right)\left(\begin{array}{cc}
1 & \mathbf{0} \\
\boldsymbol{\beta}_{i} & \mathbf{I}_{k}
\end{array}\right),
\end{gathered}
$$

$\mathbf{I}_{k}$ is an identity matrix of order $k$, and the rank of $\mathbf{C}_{i}$ is determined by the rank of the $m \times(k+1)$ matrix of the unobserved factor loadings

$$
\tilde{\Gamma}_{i}=\left(\begin{array}{cc}
\gamma_{i} & \Gamma_{i}
\end{array}\right)
$$

As discussed in Pesaran (2004), the above set up is sufficiently general and renders a variety of panel data models as special cases. In the panel literature with $T$ small and $N$ large, the primary parameters of interest are the means of the individual specific slope coefficients, $\boldsymbol{\beta}_{i}$, $i=1,2, \ldots, N$. The common factor loadings, $\boldsymbol{\alpha}_{i}$ and $\boldsymbol{\gamma}_{i}$, are generally treated as nuisance parameters. In cases where both $N$ and $T$ are large, it is also possible to consider consistent estimation of the factor loadings. The presence of the unobserved factor in (1) implies that estimation of $\boldsymbol{\beta}_{i}$ and its cross sectional mean cannot be undertaken using standard methods. Pesaran (2004) has suggested using cross section averages of $y_{i t}$ and $\mathbf{x}_{i t}$ as proxies for the unobserved factors in (1). To see why such an approach could work, consider simple cross section averages of the equations in $(3)^{1}$

$$
\overline{\mathbf{z}}_{t}=\overline{\mathbf{B}}^{\prime} \mathbf{d}_{t}+\overline{\mathbf{C}}^{\prime} \mathbf{f}_{t}+\overline{\mathbf{u}}_{t}
$$

where

$$
\overline{\mathbf{z}}_{t}=\frac{1}{N} \sum_{i=1}^{N} \mathbf{z}_{i t}, \overline{\mathbf{u}}_{t}=\frac{1}{N} \sum_{i=1}^{N} \mathbf{u}_{i t}
$$

\footnotetext{
${ }^{1}$ Pesaran (2004) considers cross section weighted averages that are more general. But to simplify the exposition we confine our discussion to simple averages throughout.
} 
and

$$
\overline{\mathbf{B}}=\frac{1}{N} \sum_{i=1}^{N} \mathbf{B}_{i}, \overline{\mathbf{C}}=\frac{1}{N} \sum_{i=1}^{N} \mathbf{C}_{i}
$$

Suppose that

$$
\operatorname{Rank}(\overline{\mathbf{C}})=m \leq k+1, \text { for all } N
$$

Then, we have

$$
\mathbf{f}_{t}=\left(\overline{\mathbf{C}} \overline{\mathbf{C}}^{\prime}\right)^{-1} \overline{\mathbf{C}}\left(\overline{\mathbf{z}}_{t}-\overline{\mathbf{B}}^{\prime} \mathbf{d}_{t}-\overline{\mathbf{u}}_{t}\right)
$$

But since

$$
\overline{\mathbf{u}}_{t} \stackrel{q . m .}{\rightarrow} \mathbf{0}, \text { as } N \rightarrow \infty, \text { for each } t,
$$

and

$$
\overline{\mathbf{C}} \stackrel{p}{\rightarrow} \mathbf{C}=\tilde{\boldsymbol{\Gamma}}\left(\begin{array}{cc}
1 & 0 \\
\boldsymbol{\beta} & \mathbf{I}_{k}
\end{array}\right), \text { as } N \rightarrow \infty
$$

where

$$
\tilde{\boldsymbol{\Gamma}}=\left(E\left(\boldsymbol{\gamma}_{i}\right), E\left(\boldsymbol{\Gamma}_{i}\right)\right)=(\boldsymbol{\gamma}, \boldsymbol{\Gamma}) .
$$

it follows, assuming that $\operatorname{Rank}(\tilde{\boldsymbol{\Gamma}})=m$, that

$$
\mathbf{f}_{t}-\left(\mathbf{C C}^{\prime}\right)^{-1} \mathbf{C}\left(\overline{\mathbf{z}}_{t}-\overline{\mathbf{B}}^{\prime} \mathbf{d}_{t}\right) \stackrel{p}{\rightarrow} \mathbf{0} \text {, as } N \rightarrow \infty
$$

This suggests using $\overline{\mathbf{h}}_{t}=\left(\mathbf{d}_{t}^{\prime}, \overline{\mathbf{z}}_{t}^{\prime}\right)^{\prime}$ as observable proxies for $\mathbf{f}_{t}$, and is the basic insight that lies behind the Common Correlated Effects estimators developed in Pesaran (2004) . It is further shown that the CCE estimation procedure in fact holds even if $\tilde{\boldsymbol{\Gamma}}$ turns out to be rank deficient.

We now discuss the two estimators for the means of the individual specific slope coefficients proposed by Pesaran (2004) . One is the Mean Group (MG) estimator proposed in Pesaran and Smith (1995) and the other is a generalization of the fixed effects estimator that allows for the possibility of cross section dependence. The former is referred to as the "Common Correlated Effects Mean Group" (CCEMG) estimator, and the latter as the "Common Correlated Effects Pooled" (CCEP) estimator.

The CCEMG estimator is a simple average of the individual CCE estimators, $\hat{\mathbf{b}}_{i}$ of $\boldsymbol{\beta}_{i}$,

$$
\hat{\mathbf{b}}_{M G}=N^{-1} \sum_{i=1}^{N} \hat{\mathbf{b}}_{i} .
$$

where

$$
\hat{\mathbf{b}}_{i}=\left(\mathbf{X}_{i}^{\prime} \overline{\mathbf{M}} \mathbf{X}_{i}\right)^{-1} \mathbf{X}_{i}^{\prime} \overline{\mathbf{M}} \mathbf{y}_{i}
$$


$\mathbf{X}_{i}=\left(\mathbf{x}_{i 1}, \mathbf{x}_{i 2}, \ldots, \mathbf{x}_{i T}\right)^{\prime}, \mathbf{y}_{i}=\left(y_{i 1}, y_{i 2}, \ldots, y_{i T}\right)^{\prime}, \overline{\mathbf{M}}$ is defined by

$$
\overline{\mathbf{M}}=\mathbf{I}_{T}-\overline{\mathbf{H}}\left(\overline{\mathbf{H}}^{\prime} \overline{\mathbf{H}}\right)^{-1} \overline{\mathbf{H}}^{\prime}
$$

$\overline{\mathbf{H}}=(\mathbf{D}, \overline{\mathbf{Z}}), \mathbf{D}$ and $\overline{\mathbf{Z}}$ being, respectively, the $T \times n$ and $T \times(k+1)$ matrices of observations on $\mathbf{d}_{t}$ and $\overline{\mathbf{z}}_{t}$.

Under certain conditions, Pesaran (2004) has shown that

$$
\sqrt{N}\left(\hat{\mathbf{b}}_{M G}-\boldsymbol{\beta}\right) \stackrel{d}{\rightarrow} N\left(\mathbf{0}, \boldsymbol{\Sigma}_{M G}\right), \text { as }(N, T) \stackrel{j}{\rightarrow} \infty
$$

where $\boldsymbol{\Sigma}_{M G}$ can be consistently estimated non-parametrically by

$$
\hat{\mathbf{\Sigma}}_{M G}=\frac{1}{N-1} \sum_{i=1}^{N}\left(\hat{\mathbf{b}}_{i}-\hat{\mathbf{b}}_{M G}\right)\left(\hat{\mathbf{b}}_{i}-\hat{\mathbf{b}}_{M G}\right)^{\prime} .
$$

Efficiency gains from pooling of observations over the cross section units can be achieved when the individual slope coefficients, $\boldsymbol{\beta}_{i}$, are the same. Such a pooled estimator of $\boldsymbol{\beta}$, denoted by CCEP, has been developed by Pesaran (2004) and is given by

$$
\hat{\mathbf{b}}_{P}=\left(\sum_{i=1}^{N} \mathbf{X}_{i}^{\prime} \overline{\mathbf{M}} \mathbf{X}_{i}\right)^{-1} \sum_{i=1}^{N} \mathbf{X}_{i}^{\prime} \overline{\mathbf{M}} \mathbf{y}_{i}
$$

Again, Pesaran (2004) has shown that

$$
N^{-1 / 2}\left(\hat{\mathbf{b}}_{P}-\boldsymbol{\beta}\right) \stackrel{d}{\rightarrow} N\left(\mathbf{0}, \boldsymbol{\Sigma}_{P}^{*}\right)
$$

where $\boldsymbol{\Sigma}_{P}^{*}$ can be consistently estimated by

$$
\widehat{A \operatorname{Var}}\left(N^{-1 / 2}\left(\hat{\mathbf{b}}_{P}-\boldsymbol{\beta}\right)\right)=\hat{\mathbf{\Psi}}^{*-1} \hat{\mathbf{R}}^{*} \hat{\mathbf{\Psi}}^{*-1} .
$$

where

$$
\hat{\mathbf{R}}^{*}=\frac{1}{N-1} \sum_{i=1}^{N}\left(\frac{\mathbf{X}_{i}^{\prime} \overline{\mathbf{M}} \mathbf{X}_{i}}{T}\right)\left(\hat{\mathbf{b}}_{i}-\hat{\mathbf{b}}_{M G}\right)\left(\hat{\mathbf{b}}_{i}-\hat{\mathbf{b}}_{M G}\right)^{\prime}\left(\frac{\mathbf{X}_{i}^{\prime} \overline{\mathbf{M}} \mathbf{X}_{i}}{T}\right)
$$

and

$$
\hat{\mathbf{\Psi}}^{*}=\frac{1}{N} \sum_{i=1}^{N}\left(\frac{\mathbf{X}_{i}^{\prime} \overline{\mathbf{M}} \mathbf{X}_{i}}{T}\right)
$$

\section{A Principal Components Augmentation Approach}

In this section we explore an alternative method of estimating the model given by (1) and

(2) based on principal component analysis as discussed in the work of Stock and Watson (2002) . Our approach is first to apply the Bai and $\mathrm{Ng}(2002)$ procedure to $\mathbf{z}_{i t}=\left(y_{i t}, \mathbf{x}_{i t}^{\prime}\right)^{\prime}$ to 
obtain consistent estimates of the unobserved factors, and then use these factor estimates to augment the regression (1), and thus produce consistent estimates of $\boldsymbol{\beta}$. A formal justification for such an approach is as follows. Recall from (3) that

$$
\mathbf{z}_{i t}=\left(\begin{array}{c}
y_{i t} \\
\mathbf{x}_{i t}
\end{array}\right)=\mathbf{\Theta}^{\prime} \mathbf{g}_{t}+\mathbf{u}_{i t}
$$

where $\boldsymbol{\Theta}_{i}^{\prime}=\left(\mathbf{B}_{i}^{\prime}, \mathbf{C}_{i}^{\prime}\right), \mathbf{g}_{t}=\left(\mathbf{d}_{t}^{\prime}, \mathbf{f}_{t}^{\prime}\right)^{\prime}$. The errors $\mathbf{u}_{i t}$, can be serially correlated but do not have common factors and are cross sectionally independent by assumption. Therefore, under Assumptions 1-5 of Pesaran (2004), this model satisfies assumptions of Bai (2003) and the common factors $\mathbf{g}_{t}$, can be consistently estimated (up to a non-singular transformation) from the principal components of $\mathbf{z}_{i t}$ for $i=1,2, \ldots, N$, and $t=1,2, \ldots, T$.

The estimated factors at time $t$, denoted by $\hat{\mathbf{f}}_{t}$ will be linear combinations of the $(m+n) \times 1$ vector $\mathbf{g}_{t}$. It is important to note that $m+n$, rather than just $m$ factors, must be extracted from $\mathbf{z}_{i t}$. In practice, $m$ is not known and must be replaced by an estimate using the selection procedure in Bai and $\mathrm{Ng}$ (2002), for example. This in turn can introduce a certain degree of sampling uncertainty into the analysis.

Once these factors are extracted we can use the results of Bai (2003) and in particular Comment 2 (pp. 146) to justify augmenting (1) by the estimated factors. In particular, Bai (2003) shows that as long as $\sqrt{T} / N \rightarrow 0$ the error in the estimated factor is negligible for estimating the regression

$$
y_{i t}=\boldsymbol{\alpha}_{i}^{\prime} \mathbf{d}_{t}+\boldsymbol{\beta}_{i}^{\prime} \mathbf{x}_{i t}+\boldsymbol{\gamma}_{i}^{\prime} \hat{\mathbf{f}}_{t}+\eta_{i t}
$$

Again we consider both mean group and pooled estimators. The mean group and pooled estimators are given respectively by

$$
\hat{\mathbf{b}}_{M G P C}=N^{-1} \sum_{i=1}^{N} \hat{\mathbf{b}}_{M G P C, i}
$$

and

where

$$
\hat{\mathbf{b}}_{P P C}=\left(\sum_{i=1}^{N} \mathbf{X}_{i}^{\prime} \mathbf{M}_{\hat{g}} \mathbf{X}_{i}\right)^{-1} \sum_{i=1}^{N} \mathbf{X}_{i}^{\prime} \mathbf{M}_{\hat{g}} \mathbf{y}_{i}
$$

$$
\mathbf{M}_{\hat{g}}=\mathbf{I}_{T}-\hat{\mathbf{G}}\left(\hat{\mathbf{G}}^{\prime} \hat{\mathbf{G}}\right)^{-1} \hat{\mathbf{G}}^{\prime},
$$

$\hat{\mathbf{G}}=(\mathbf{D}, \hat{\mathbf{F}}), \hat{\mathbf{F}}$ is the $T \times(m+n)$ matrix of observations on $\hat{\mathbf{f}}_{t}$, and $\hat{\mathbf{b}}_{M G P C, i}$ is the estimator of $\boldsymbol{\beta}_{i}$ in (23). The variance for the mean group estimator is given by

$$
A \hat{V a r}\left(\hat{\mathbf{b}}_{M G P C}\right)=\frac{1}{N-1} \sum_{i=1}^{N}\left(\hat{\mathbf{b}}_{M G P C, i}-\hat{\mathbf{b}}_{M G P C}\right)\left(\hat{\mathbf{b}}_{M G P C, i}-\hat{\mathbf{b}}_{M G P C}\right)^{\prime}
$$


and for the pooled estimator, in the case where $\boldsymbol{\beta}_{i}=\boldsymbol{\beta}$, by

$$
A \hat{V} \operatorname{ar}\left(\hat{\mathbf{b}}_{P P C}\right)=\frac{1}{N}\left(\sum_{i=1}^{N} \mathbf{X}_{i}^{\prime} \mathbf{M}_{\hat{g}} \mathbf{X}_{i}\right)^{-1}\left(\sum_{i=1}^{N} \hat{\sigma}_{i}^{2} \mathbf{X}_{i}^{\prime} \mathbf{M}_{\hat{g}} \mathbf{X}_{i}\right)\left(\sum_{i=1}^{N} \mathbf{X}_{i}^{\prime} \mathbf{M}_{\hat{g}} \mathbf{X}_{i}\right)^{-1}
$$

where $\hat{\sigma}_{i}^{2}$ is the estimated error variance of $(23)$.

The principal components are computed based on standardized observations, namely $\left(y_{i t}-\bar{y}_{i}\right) / s_{i}$ and $\left(x_{i t \ell}-\bar{x}_{i \ell}\right) / s_{i l}$ where $\bar{y}_{i}$ and $\bar{x}_{i \ell}$ are sample means of $y_{i t}$ and the $\ell^{\text {th }}$ element of $\mathbf{x}_{i t}$, and $s_{i}$ and $s_{i \ell}$ are the associated sample standard deviations.

\section{Small Sample Properties of the Various Estimators}

\subsection{Monte Carlo Design}

The data generating processes used in the Monte Carlo experiments are different parameterizations of (1) and (2) which we reproduce here for convenience:

$$
y_{i t}=\boldsymbol{\alpha}_{i}^{\prime} \mathbf{d}_{t}+\boldsymbol{\beta}_{i}^{\prime} \mathbf{x}_{i t}+\boldsymbol{\gamma}_{i}^{\prime} \mathbf{f}_{t}+\varepsilon_{i t},
$$

and

$$
\mathbf{x}_{i t}=\mathbf{A}_{i}^{\prime} \mathbf{d}_{t}+\boldsymbol{\Gamma}_{i}^{\prime} \mathbf{f}_{t}+\mathbf{v}_{i t}
$$

where $\mathbf{A}_{i}=\left[\alpha_{i s l}\right]$ and $\boldsymbol{\Gamma}_{i}=\left[\gamma_{i s l}\right]$ are $n \times k$ and $m \times k$, factor loading matrices with fixed components, $\mathbf{v}_{i t}$ are the specific components of $\mathbf{x}_{i t}$ distributed independently of the common effects and across $i$, but assumed to follow general covariance stationary processes.

In the calibration of the Monte Carlo design, it is important that the population value of $R^{2}$ for (29) is controlled across the different experiments. Otherwise, comparisons of the power properties of the different estimators can be misleading in the case of models with different numbers of observed and unobserved factors. In what follows we show how the average population $R^{2}$ of the $y_{i t}$ equation varies with the model parameter and hence find values of the error variances, $\sigma_{i}^{2}$, that ensure the population $R^{2}$ is around $60 \%$ irrespective of the number of regressors included in the model. We shall assume that unconditionally

$$
\begin{aligned}
\mathbf{f}_{t} & \sim\left(\mathbf{0}, \mathbf{\Sigma}_{f}\right), \mathbf{d}_{t} \sim\left(\mathbf{0}, \mathbf{\Sigma}_{d}\right) \\
\varepsilon_{i t} & \sim \operatorname{IID}\left(0, \sigma_{i}^{2}\right), \mathbf{v}_{i t} \sim \operatorname{IID}\left(\mathbf{0}, \mathbf{\Sigma}_{v i}\right) .
\end{aligned}
$$

The variables, $\mathbf{f}_{t}, \mathbf{d}_{t}, \varepsilon_{i t}, \mathbf{v}_{i t}$ are also assumed to be distributed independently of the parameters, $\boldsymbol{\alpha}_{i}, \boldsymbol{\beta}_{i}, \boldsymbol{\Gamma}_{i}, \mathbf{A}_{i}$, and $\boldsymbol{\gamma}_{i}$. Using (30) in (29) we have

$$
\begin{aligned}
& y_{i t}=\boldsymbol{\alpha}_{i}^{\prime} \mathbf{d}_{t}+\boldsymbol{\beta}_{i}^{\prime}\left(\mathbf{A}_{i}^{\prime} \mathbf{d}_{t}+\boldsymbol{\Gamma}_{i}^{\prime} \mathbf{f}_{t}+\mathbf{v}_{i t}\right)+\boldsymbol{\gamma}_{i}^{\prime} \mathbf{f}_{t}+\varepsilon_{i t}, \\
& y_{i t}=\boldsymbol{\varphi}_{i}^{\prime} \mathbf{g}_{t}+e_{i t},
\end{aligned}
$$


where

$$
\boldsymbol{\varphi}_{i}=\left(\begin{array}{c}
\boldsymbol{\alpha}_{i}+\mathbf{A}_{i} \boldsymbol{\beta}_{i} \\
\gamma_{i}+\boldsymbol{\Gamma}_{i} \boldsymbol{\beta}_{i}
\end{array}\right), \mathbf{g}_{t}=\left(\begin{array}{c}
\mathbf{d}_{t} \\
\mathbf{f}_{t}
\end{array}\right)
$$

and

$$
e_{i t}=\varepsilon_{i t}+\boldsymbol{\beta}_{i}^{\prime} \mathbf{v}_{i t} .
$$

The population $R^{2}$ of (29), conditional on $\boldsymbol{\varphi}_{i}$ and $\boldsymbol{\beta}_{i}$, is given by

$$
R_{i}^{2}=1-\frac{\sigma_{i}^{2}}{\operatorname{Var}\left(y_{i t} \mid \boldsymbol{\varphi}_{i}, \boldsymbol{\beta}_{i}\right)},
$$

where

$$
\operatorname{Var}\left(y_{i t} \mid \boldsymbol{\varphi}_{i}, \boldsymbol{\beta}_{i}\right)=\boldsymbol{\varphi}_{i}^{\prime} \boldsymbol{\Sigma}_{g} \boldsymbol{\varphi}_{i}+\boldsymbol{\beta}_{i}^{\prime} \boldsymbol{\Sigma}_{v i} \boldsymbol{\beta}_{i}+\sigma_{i}^{2},
$$

and

$$
\Sigma_{g}=\left(\begin{array}{cc}
\Sigma_{d} & \Sigma_{d f} \\
\Sigma_{f d} & \Sigma_{f}
\end{array}\right)
$$

Since, $E\left(y_{i t} \mid \boldsymbol{\varphi}_{i}, \boldsymbol{\beta}_{i}\right)=0$, then on average (integrating out the individual effects)

$$
\begin{aligned}
& \operatorname{Var}\left(y_{i t}\right)=E\left(\boldsymbol{\varphi}_{i}^{\prime} \boldsymbol{\Sigma}_{g} \boldsymbol{\varphi}_{i}+\boldsymbol{\beta}_{i}^{\prime} \boldsymbol{\Sigma}_{v i} \boldsymbol{\beta}_{i}+\sigma_{i}^{2}\right), \\
& \operatorname{Var}\left(\varepsilon_{i t}\right)=E\left(\sigma_{i}^{2}\right),
\end{aligned}
$$

and the average population $R^{2}$ value will be given by

$$
R^{2}=1-\frac{E\left(\sigma_{i}^{2}\right)}{E\left(\boldsymbol{\varphi}_{i}^{\prime} \boldsymbol{\Sigma}_{g} \boldsymbol{\varphi}_{i}+\boldsymbol{\beta}_{i}^{\prime} \boldsymbol{\Sigma}_{v i} \boldsymbol{\beta}_{i}+\sigma_{i}^{2}\right)} .
$$

Suppose that the individual-specific parameters, $\sigma_{i}^{2}, \boldsymbol{\varphi}_{i}$ and $\boldsymbol{\beta}_{i}$, are distributed independently of $\boldsymbol{\Sigma}_{g}$, and $\boldsymbol{\beta}_{i}$ is distributed independently of $\boldsymbol{\Sigma}_{v i}$ with constant means and variances, $\sigma^{2}=E\left(\sigma_{i}^{2}\right), \boldsymbol{\varphi}=E\left(\boldsymbol{\varphi}_{i}\right), \boldsymbol{\beta}=E\left(\boldsymbol{\beta}_{i}\right), \operatorname{Var}\left(\boldsymbol{\varphi}_{i}\right)=\boldsymbol{\Sigma}_{\boldsymbol{\varphi}}, \operatorname{Var}\left(\boldsymbol{\beta}_{i}\right)=\boldsymbol{\Sigma}_{\boldsymbol{\beta}}$, and $\boldsymbol{\Sigma}_{v}=E\left(\boldsymbol{\Sigma}_{v i}\right)$.

Then it is easily seen that

$$
R^{2}=1-\frac{\sigma^{2}}{\boldsymbol{\varphi}^{\prime} \boldsymbol{\Sigma}_{g} \boldsymbol{\varphi}+\operatorname{Tr}\left(\boldsymbol{\Sigma}_{g} \boldsymbol{\Sigma}_{\boldsymbol{\varphi}}\right)+\boldsymbol{\beta}^{\prime} \boldsymbol{\Sigma}_{v} \boldsymbol{\beta}+\operatorname{Tr}\left(\boldsymbol{\Sigma}_{v} \boldsymbol{\Sigma}_{\boldsymbol{\beta}}\right)+\sigma^{2}},
$$

where $\boldsymbol{\Sigma}_{\boldsymbol{\varphi}}=E\left(\boldsymbol{\varphi}_{i} \boldsymbol{\varphi}_{i}^{\prime}\right)-\boldsymbol{\varphi} \boldsymbol{\varphi}^{\prime}$. To derive $E\left(\boldsymbol{\varphi}_{i} \boldsymbol{\varphi}_{i}^{\prime}\right)$, let $\mathbf{A}_{i}^{\prime}=\left(\mathbf{a}_{i 1}, \mathbf{a}_{i 2}, \ldots, \mathbf{a}_{i n}\right), \boldsymbol{\gamma}_{i}^{\prime}=\left(\boldsymbol{\gamma}_{i 1}, \boldsymbol{\gamma}_{i 2}, \ldots, \boldsymbol{\gamma}_{i m}\right)$ and note that

$$
\boldsymbol{\varphi}_{i} \boldsymbol{\varphi}_{i}^{\prime}=\left(\begin{array}{cc}
\left(\boldsymbol{\alpha}_{i}+\mathbf{A}_{i} \boldsymbol{\beta}_{i}\right)\left(\boldsymbol{\alpha}_{i}+\mathbf{A}_{i} \boldsymbol{\beta}_{i}\right)^{\prime} & \left(\boldsymbol{\alpha}_{i}+\mathbf{A}_{i} \boldsymbol{\beta}_{i}\right)\left(\boldsymbol{\gamma}_{i}+\boldsymbol{\Gamma}_{i} \boldsymbol{\beta}_{i}\right)^{\prime} \\
\left(\boldsymbol{\gamma}_{i}+\boldsymbol{\Gamma}_{i} \boldsymbol{\beta}_{i}\right)\left(\boldsymbol{\alpha}_{i}+\mathbf{A}_{i} \boldsymbol{\beta}_{i}\right)^{\prime} & \left(\boldsymbol{\gamma}_{i}+\boldsymbol{\Gamma}_{i} \boldsymbol{\beta}_{i}\right)\left(\boldsymbol{\gamma}_{i}+\boldsymbol{\Gamma}_{i} \boldsymbol{\beta}_{i}\right)^{\prime}
\end{array}\right),
$$

$$
\begin{aligned}
E\left(\mathbf{A}_{i} \boldsymbol{\beta}_{i} \boldsymbol{\beta}_{i}^{\prime} \mathbf{A}_{i}^{\prime}\right) & =\left(\begin{array}{cccc}
E\left(\boldsymbol{\beta}_{i}^{\prime} \mathbf{a}_{i 1} \mathbf{a}_{i 1}^{\prime} \boldsymbol{\beta}_{i}\right) & E\left(\boldsymbol{\beta}_{i}^{\prime} \mathbf{a}_{i 1} \mathbf{a}_{i 2}^{\prime} \boldsymbol{\beta}_{i}\right) & E\left(\boldsymbol{\beta}_{i}^{\prime} \mathbf{a}_{i 1} \mathbf{a}_{i n}^{\prime} \boldsymbol{\beta}_{i}\right) \\
E\left(\boldsymbol{\beta}_{i}^{\prime} \mathbf{a}_{i 1} \mathbf{a}_{i 2}^{\prime} \boldsymbol{\beta}_{i}\right) & E\left(\boldsymbol{\beta}_{i}^{\prime} \mathbf{a}_{i 2} \mathbf{a}_{i 2}^{\prime} \boldsymbol{\beta}_{i}\right) & E\left(\boldsymbol{\beta}_{i}^{\prime} \mathbf{a}_{i 2} \mathbf{a}_{i n}^{\prime} \boldsymbol{\beta}_{i}\right) \\
\vdots & \vdots & \vdots & \vdots \\
E\left(\boldsymbol{\beta}_{i}^{\prime} \mathbf{a}_{i n} \mathbf{a}_{i 1}^{\prime} \boldsymbol{\beta}_{i}\right) & \cdots & & E\left(\boldsymbol{\beta}_{i}^{\prime} \mathbf{a}_{i n} \mathbf{a}_{i n}^{\prime} \boldsymbol{\beta}_{i}\right)
\end{array}\right) .
\end{aligned}
$$


Assuming $\boldsymbol{\beta}_{i}$ is distributed independently of $\mathbf{a}_{i r}$ for $r=1,2, . ., n$, we have

$$
E\left(\boldsymbol{\beta}_{i}^{\prime} \mathbf{a}_{i r} \mathbf{a}_{i s}^{\prime} \boldsymbol{\beta}_{i}\right)=\boldsymbol{\beta}^{\prime} E\left(\mathbf{a}_{i r} \mathbf{a}_{i s}^{\prime}\right) \boldsymbol{\beta}+\operatorname{Tr}\left[E\left(\mathbf{a}_{i r} \mathbf{a}_{i s}^{\prime}\right) \boldsymbol{\Sigma}_{\beta}\right]
$$

for $r, s=1,2, \ldots, n$. Similarly, assuming $\boldsymbol{\beta}_{i}$ is distributed independently of $\boldsymbol{\gamma}_{i r}$

$$
E\left(\boldsymbol{\beta}_{i}^{\prime} \boldsymbol{\gamma}_{i r} \boldsymbol{\gamma}_{i s}^{\prime} \boldsymbol{\beta}_{i}\right)=\boldsymbol{\beta}^{\prime} E\left(\boldsymbol{\gamma}_{i r} \gamma_{i s}^{\prime}\right) \boldsymbol{\beta}+\operatorname{Tr}\left[E\left(\boldsymbol{\gamma}_{i r} \boldsymbol{\gamma}_{i s}^{\prime}\right) \boldsymbol{\Sigma}_{\beta}\right]
$$

for $r, s=1,2, \ldots, m$. The remaining elements of $E\left(\boldsymbol{\varphi}_{i} \boldsymbol{\varphi}_{i}^{\prime}\right)$ can also be obtained in a similar fashion.

For the Monte Carlo experiments we used the following parameterizations

$$
\begin{gathered}
\boldsymbol{\Sigma}_{\boldsymbol{\beta}}=\mathbf{0}, \boldsymbol{\Sigma}_{g}=\left(\begin{array}{cc}
\mathbf{I}_{n} & \mathbf{0} \\
\mathbf{0} & \boldsymbol{\Sigma}_{f}
\end{array}\right), \boldsymbol{\Sigma}_{v}=\mathbf{I}_{k} \\
\boldsymbol{\Sigma}_{f}=\left(\begin{array}{cccc}
1 & \theta & \cdots & \theta \\
\theta & 1 & \cdots & \theta \\
\vdots & \vdots & \vdots & \vdots \\
\theta & \theta & \cdots & 1
\end{array}\right)
\end{gathered}
$$

$\theta$ is the pair-wise correlation coefficient of the unobserved factors and

$$
\varphi=\left(\begin{array}{c}
\alpha+\mathbf{A} \beta \\
\gamma+\boldsymbol{\Gamma} \boldsymbol{\beta}
\end{array}\right)
$$

Also since the parameters are generated independently we have

$$
\begin{aligned}
E\left(\boldsymbol{\alpha}_{i} \boldsymbol{\beta}_{i}^{\prime} \mathbf{A}_{i}^{\prime}\right) & =\boldsymbol{\alpha} \boldsymbol{\beta}^{\prime} \mathbf{A}^{\prime}, \\
E\left(\boldsymbol{\alpha}_{i} \boldsymbol{\beta}_{i}^{\prime} \boldsymbol{\Gamma}_{i}^{\prime}\right) & =\boldsymbol{\alpha} \boldsymbol{\beta}^{\prime} \boldsymbol{\Gamma}^{\prime},
\end{aligned}
$$

and

$$
\begin{aligned}
E\left(\boldsymbol{\alpha}_{i} \boldsymbol{\alpha}_{i}^{\prime}\right) & =\boldsymbol{\Sigma}_{\alpha}+\boldsymbol{\alpha} \boldsymbol{\alpha}^{\prime} \\
E\left(\boldsymbol{\gamma}_{i} \boldsymbol{\gamma}_{i}^{\prime}\right) & =\boldsymbol{\Sigma}_{\gamma}+\boldsymbol{\gamma} \boldsymbol{\gamma}^{\prime} . \\
E\left(\mathbf{a}_{i r} \mathbf{a}_{i s}^{\prime}\right) & =\mathbf{a}_{r} \mathbf{a}_{s}^{\prime} \text { if } r \neq s \\
& =\Sigma_{a_{r}}+\mathbf{a}_{r} \mathbf{a}_{r}^{\prime},
\end{aligned}
$$

assuming that $\mathbf{a}_{i r}$ are identically distributed. Similarly,

$$
\begin{aligned}
E\left(\gamma_{i r} \gamma_{i s}^{\prime}\right) & =\gamma_{r} \gamma_{s}^{\prime} \text { if } r \neq s \\
& =\Sigma_{\gamma_{r}}+\gamma_{r} \gamma_{r}^{\prime}
\end{aligned}
$$

Using the above framework we carried out two different sets of experiments. In the first set, which we denote by A, we consider the small sample properties of the CCE and PC 
augmented estimators. In a second set, denoted as experiments $\mathrm{B}$, we also investigate the small sample properties of estimators obtained from regressing $y_{i t}$ on $\mathbf{d}_{t}, \mathbf{x}_{i t}$ and a sub-set of $\mathbf{f}_{t}$ obtained using two different information criteria, namely the Akaike type criterion where the objective function to be minimized is given by

$$
I C_{1}(f)=\frac{T}{2} \sum_{i=1}^{N} \ln \left(\hat{\varepsilon}_{i}^{\prime} \hat{\varepsilon}_{i} / T\right)+N K
$$

where $\hat{\varepsilon}_{i}$ are the residuals of (29) for cross sectional unit $i$, and $K$ denotes the total number of regressors in(29). The second criterion is a Theil type criterion defined by

$$
I C_{2}(f)=\frac{\frac{T}{2} \sum_{i=1}^{N} \ln \left(\hat{\varepsilon}_{i}^{\prime} \hat{\varepsilon}_{i} / T\right)}{N\left(T-m_{y}\right)-k}
$$

where $m_{y}<m$ denotes the number of factors entering (29). The criteria are minimized over all possible factor combinations. These experiments are intended to highlight the dependence of the PC augmented procedure on the choice of the factors, even if satisfactory estimates of $\mathbf{f}_{t}$ can be obtained using the PC procedure.

For all experiments $T, N=30,50,100,200, n=3, \boldsymbol{\beta}=(1,1, \ldots, 1)^{\prime}$. For experiments A, $k=1,3$. For experiments B, $k=1$. Partition $\mathbf{d}_{t}$ as follows: $\mathbf{d}_{t}=\left(d_{1 t}, d_{2 t}, d_{3 t}\right)^{\prime}$ and partition conformably $\boldsymbol{\alpha}_{i}$ and $\mathbf{A}_{i} . d_{1 t}=1$. For experiments A, $m=1,2,3,4$. For experiments B, $m=5$. Further, we set

$$
\begin{gathered}
\boldsymbol{\alpha}_{i}=\left(\alpha_{i 1}, \alpha_{i 2}, \ldots, \alpha_{i k+1}, 0, \ldots, 0\right)^{\prime}, \alpha_{i j} \sim \operatorname{IIDU}(0.5,1.5), j=1, \ldots, k+1 \\
\alpha_{i s l}=0 ; s=1, \ldots, n ; l=1, k+1 \\
\alpha_{i s l} \sim U(0.5,1.5) ; s=1, \ldots, n ; l=k+2, \ldots, n
\end{gathered}
$$

$d_{j t}$ is given by

$$
d_{j t}=\rho_{d_{j}} d_{j t-1}+\varepsilon_{d_{j} t}, j=2, \ldots, n
$$

where $\rho_{d_{j}}=0.4, j=2, \ldots, n$ and $\varepsilon_{d_{j} t} \sim \operatorname{IIDN}\left(0,1-\rho_{d_{j}}^{2}\right) . \gamma_{i}=\left(\gamma_{1 i}, \ldots, \gamma_{m i}\right)^{\prime}$ where $\gamma_{j i} \sim$ $\operatorname{IIDN}(1,0.04)$ for experiment A. For experiment B $\gamma_{j i} \sim \operatorname{IIDN}(1,0.04), j=1,2,3$ and $\gamma_{j i}=0$ for $j=4,5$.

$$
\gamma_{i s l} \sim \operatorname{IIDU}(0.5,1.5)
$$

$\mathbf{v}_{i t}$ is given by

$$
\mathbf{v}_{i t}=\Phi_{i} \mathbf{v}_{i t-1}+\varepsilon_{i \mathbf{v} t}
$$


where $\Phi_{i}=\operatorname{diag}\left(\rho_{v 1 i}, \ldots, \rho_{v k i}\right), \varepsilon_{i \mathbf{v} t} \sim N\left(0, \boldsymbol{\Sigma}_{i v}\right), \rho_{v j i} \sim U(0.2,0.9), \boldsymbol{\Sigma}_{i v}=\operatorname{diag}\left(1-\rho_{v 1 i}^{2}, \ldots, 1-\right.$ $\left.\rho_{v k i}^{2}\right)$. Finally, $\mathbf{f}_{t}=\left(f_{1 t}, \ldots, f_{m t}\right)^{\prime}$

$$
f_{j t}=\rho_{f_{j}} f_{j t-1}+\varepsilon_{f_{j} t}, j=2, \ldots, m
$$

where $\rho_{d_{j}} \sim \operatorname{IIDU}(0.2,0.9), j=2, \ldots, m, \varepsilon_{f_{j} t}=\sqrt{1-\rho_{f_{j}}^{2}} \omega_{j t}, \omega_{j t}=\sqrt{\theta} \omega_{t}+(1-\theta) \varpi_{j t}$ and $\omega_{t}, \varpi_{j t} \sim N(0,1)$. Hence, $\operatorname{Var}\left(f_{i t}\right)=1$, and $\operatorname{Corr}\left(f_{i t}, f_{j t}\right)=\theta$, as required.

To ensure a constant average $R^{2}$ of around 0.6 for all experiments we generated the equation-specific errors according to

$$
\varepsilon_{i t} \sim \operatorname{IIDN}\left(0, \sigma_{i}^{2}\right), \sigma_{i}^{2} \sim \operatorname{IID} \frac{h(k, m)}{2} \chi_{2}^{2},
$$

where the scaler $h(k, m)$ is set in terms of $m$ and $k$ as

\begin{tabular}{|lll|}
\hline$m$ & $k$ & $h(k, m)$ \\
\hline 1 & 1 & 8 \\
1 & 2 & 16 \\
1 & 3 & 26 \\
2 & 1 & 11 \\
2 & 2 & 32 \\
2 & 3 & 53 \\
3 & 1 & 22 \\
3 & 2 & 48 \\
3 & 3 & 74 \\
4 & 1 & 29 \\
4 & 2 & 70 \\
4 & 3 & 155 \\
5 & 1 & 40 \\
5 & 2 & 100 \\
5 & 3 & 190 \\
\hline
\end{tabular}

Finally, all parameters are set at the beginning of each experiment and 2000 replications are run. The only exception to that is $\gamma_{i}$ for which new parameter draws occur for every replication.

\subsection{Alternative Estimators Considered}

In the case of experiments $\mathrm{A}$, we considered four different types of estimators. First, a misspecified procedure that ignores the common unobserved effects and for efficiency purposes considers a pooled estimator under the slope homogeneity assumption, $\beta=\beta_{i}$. We denote this as the 'Naive' estimator. Second, we consider the CCEMG and CCEP estimators defined by (14) and (19). Third, we consider the PC augmented estimators defined by (24) and (25), which we denote by $M G P C$ and $P P C$, respectively. Finally, we consider the principal 
component estimator proposed by Coakley, Fuertes, and Smith (2002). For this estimator the following steps are taken. We estimate (1), using a standard pooled estimator for $\boldsymbol{\beta}_{i}$, without proxying for the factors. Then, we obtain the residuals from (1) normalise them and extract $m+n$ factors from them. We use these factors in (23) and obtain the relevant Mean Group and Pooled estimators. These are denoted MGCFS and PCFS.

As noted earlier, the estimators under experiments B, assume that all the factors are observed. In one case, there is uncertainty as to which factors enter (29) as opposed to (30). The criteria described earlier are used to select the set of factors to be included in (29). All possible combinations are considered. These are infeasible estimators since the factors are not actually observed. However, they serve the purposes of showing that using principal components does not only introduce a factor estimation problem but a model selection problem as well, which is not present with the CCE estimators. These estimators are denoted by $M G(I C)$ and $P(I C)$ where $I C$ stands for either Theil or AIC type criteria. Finally, we consider the infeasible estimators where both the factors, and the identities of the factors entering (29), are known. These estimators are denoted by MGT and PT, respectively. We present results on the Bias $(\times 10000)$ and $\operatorname{RMSE}(\times 100)$ for all these estimators. We also provide size and power estimates of the different estimators for testing the hypothesis that $\boldsymbol{\beta}=1$. The power of the tests are computed under the alternative $\boldsymbol{\beta}=1.05$.

Compared to the Monte Carlo study reported in Coakley, Fuertes, and Smith (2004), our design allows for the failure of the rank condition, (9), and provides a comparative analysis of the CCEMG and CCEP estimators. Coakley, Fuertes, and Smith (2004) find that the $C C E M G$ performs best across a number of alternative estimators. However, they do not consider the $C C E P$ estimator, although their Monte Carlo design imposes the homogeneity restrictions $\boldsymbol{\beta}_{i}=\boldsymbol{\beta}$. We also consider a more extensive analysis of multifactor and multiregressor models since we consider models of up to fours factors in conjunction with up to 3 individual specific regressors. Further, we control for the $R^{2}$ of the models which as explained earlier is of great importance for the validity of the Monte Carlo analysis. Fourthly, we consider new principal component based estimators. These estimators take into account the possibility that (30) contains unobserved common components not contained in (29) by extracting the number of factors in both (30) and (29) rather than just (29) as discussed in the previous section. More generally, via experiments B, we explore the important issue of factor selection when principal components are used for the estimation of the common effects. 


\subsection{Monte Carlo Results}

The results for experiment A are summarized in Tables 1-7, and for experiments B are given in Tables 8 and 9. It is clear from Table 1 that the 'Naive' estimator that ignores the unobserved common effects is substantially biased and over-rejects the null hypothesis often by 80-95\% margin! Considering the estimators that attempt to account for the presence of the unobserved common effects, we first note that cross section averages work as expected for the case of a single unobserved common factor. Tests using both the MG and PC estimators are correctly sized reflecting the fact that the estimated variance is a consistent estimate of the true variance. Further, the $C C E M G$ estimator has marginally worse RMSEs than the $C C E P$ estimator as expected given the efficiency gains of pooling. The improvement when $C C E P$ is used is of the order of $10 \%-15 \%$. When we move to experiments with more than one unobserved common factors similar conclusions are reached concerning the $C C E M G$ and $C C E P$ estimators. The most obvious difference relates to the size of the test that $\boldsymbol{\beta}=1$ under the null hypothesis. For both estimators there are cases where the test over-rejects slightly under the null. Most of these cases, however, relate to small $N$ experiments and the over-rejection disappears as $N$ is increased. Also, both the bias and RMSE of the estimators increase as more factors are introduced. For comparable population $R^{2}$, the power of the tests based on CCEMG and CCEP estimators also tend to decline as the number of unobserved factors are increased. This is due to the fact that the cross section averages, $\bar{y}_{t}$ and $\bar{x}_{t}$, capture smaller proportion of the time variations of the unobserved factors as the number of unobserved factors is increased. This feature could not have been observed if the population $R^{2}$ had not been kept fixed across the different experiments.

Moving on to the PC augmented estimators we note that they perform considerably worse than the $C C E$ type estimators. The biases are dramatically larger. Although the bias is reduced with increased sample sizes (both $N$ and $T$ ) they still remain much larger than the bias observed for the cross sectional average estimators. The RMSEs also tell the same story. Both $M G P C$ and $P P C$ perform badly, and substantially over-reject the null hypothesis. The MGCFS and PCFS estimators perform considerably worse that the other PC augmented estimators.

Moving on to Experiments B we note several striking features. The cross sectional average estimators work as expected. They have relatively small biases and RMSE. The size performance is reasonable even for 5 factors. But the estimators where the true factors are known but the identity of the factors entering (29) is not and needs to be determined via a selection criterion, are performing rather poorly. Further, they do not seem to improve when $N$ 
grows, but only when $T$ grows. Of course, for large $T$, they outperform the $C C E$ estimators since the factors get selected perfectly. This can be seen if one compares the result for the $M G T$ and PT estimators that include the true factors. This feature is shared by both pooled and mean group estimators. We also note that the Akaike-type criterion performs better in selecting the appropriate factors compared to the Theil-type criterion. This is the case both for the $C C E P$ and $C C E M G$ estimators. Overall, it appears that even if one knows the factors, the small sample bias in the model selection aspect of the PC augmented procedure is important enough to adversely affect the performance of the estimators for moderate values of $T$, even if one abstracts from the small sample bias in estimation of the unobserved factors.

\section{An Empirical Application}

In this section we present the results of an empirical application to a panel data set of company returns to the following stock return equations

$$
y_{i t}=\alpha_{i 1}+\alpha_{i 2} \pi_{o t}+\mathbf{x}_{i t}^{\prime} \boldsymbol{\beta}+\mathbf{f}_{t}^{\prime} \gamma_{i}+\varepsilon_{i t}
$$

where here $y_{i t}$ denotes the individual company stock returns, $\pi_{o t}$ is the rate of change of oil prices in US Dollars (representing the observed common factor of the model), $\mathbf{x}_{i t}$ is a vector of observed macroeconomic factors $\mathbf{x}_{i t}=\left(\Delta q_{i t}, \Delta \pi_{i t}, \Delta r_{i t}, \Delta m_{i t}, \Delta e_{i t}\right)^{\prime}$, where $q_{i t}$ is (log of) real output of the country of company $i$ at time $t, \pi_{i t}$ is the inflation rate of the country of company $i$ at time $t, r_{i t}$ is the real interest rate of the country of company $i$ at time $t, m_{i t}$ is the real money supply of the country of company $i$ at time $t$, and $e_{i t}$ is the real exchange rate of the country of company $i$ at time $t$ with respect to the U.S. dollar. The model is also assumed to contain $m$ unobserved common effects, $\mathbf{f}_{t}$ that could be correlated with $\mathbf{x}_{i t}$ and/or $\pi_{o t}$. We also assume that $m$ is fixed but unknown. This model is clearly a generalization of a standard APT model. It allows individual stock returns to be affected both by observed macroeconomic variables and by unobserved common factors. We report results for subsets of the above macroeconomic explanatory variables as well.

The data set contains 243 companies from France, Germany, Italy, Japan, the UK, the US, South East Asia, the Middle East and Latin America. The sample periods differ across companies and cover the period 1979Q1-1999Q1. Table A below gives details of the geographical coverage of the companies included in the panel and the associated sample periods. 


\begin{tabular}{l|cc}
\hline \multicolumn{3}{c}{ Table A: Number of Companies and the Sample Periods } \\
\hline \multicolumn{1}{c}{ Region } & \# Companies & Sample Periods \\
\hline US & 63 & $79 Q 1-99 Q 1$ \\
UK & 24 & 79Q1-99Q1 \\
Germany & 21 & 79Q1-99Q1 \\
France & 14 & 79Q1-99Q1 \\
Italy & 10 & $79 Q 1-99 Q 1$ \\
W. Europe & 24 & 79Q1-99Q1 \\
Middle East & 4 & $90 Q 3-99 Q 1$ \\
S. E. Asia & 34 & 89Q3-99Q1 \\
Japan & 35 & $79 Q 1-99 Q 1$ \\
L. America & 14 & 89Q3-99Q1 \\
\hline Total & 243 & \\
\hline
\end{tabular}

Notes: Western Europe is made up of Spain, The Netherlands, Belgium and Switzerland. The Middle East contains firms from Turkey. South East Asia contains firms from Indonesia, Korea, Malaysia, Philippines, Singapore and Thailand. Finally, Latin America contains firms from Argentina, Brazil, Chile, Mexico and Peru.

The source of the company data is Datastream, with more details provided in Pesaran, Schuermann, and Treutler (2004) . The macroeconomic data are the same as those in Pesaran, Schuermann, and Weiner (2004) and we refer the reader to that paper for details. Note that the structure of the panel data set is very rich. 10 different regions of the world are considered where each region is represented by at least 10 companies each (with the exception of Middle East). The macroeconomic variables for each company in a given region are the same. Therefore, the model in (33), can be viewed as a mixture of an APT model with observed regional macroeconomic factors and an APT model with global unobserved common effects represented by the unobserved factors and proxied by the global cross-sectional averages used by the $C C E M G$ and $C C E P$ estimators.

There is reduced coverage over time for some countries and companies. As a result this is an unbalanced panel and so our estimation methods must be modified to address this. The $C C E M G$ estimator is readily modified. The CCEP estimator is in this case still given by (19) but the matrices $\mathbf{X}_{i}^{\prime} \mathbf{y}_{i}$ and $\overline{\mathbf{M}}$ are defined so as to include only available observations for the $i$-th unit. In the unbalanced panel case the dimension of the $\overline{\mathbf{M}}$ now depends on $i$.

We report the coefficient estimates, and the test results for the null hypothesis that the coefficients are equal to zero, in Tables 10-13. Tables 10-11 report results for the pooled $(C C E P)$ estimators. Tables $12-13$ report the results for the MG estimators $(C C E M G)$. We consider a number of specifications whereby subsets of the explanatory variables are dropped 
from the regression to investigate the effect of such omissions on the remaining coefficients.

The coefficients of the $\mathbf{x}_{i t}$ variables have the expected signs and are for the most part significantly different from zero. One exception is the coefficient of the inflation variable which is generally found to be insignificant. The oil price variable does not seem to be statistically significant either. This could be due to the highly heterogeneous nature of the effect of oil prices on different company returns, being positive for companies with large oil interests such as oil or petrochemical companies, and being negative on those with significant dependence on oil, such as airlines or automobile industries. Overall, the $C C E P$ and the $C C E M G$ estimators yield very similar results, the exception being the coefficient of the interest rate variable which is much larger (in absolute value) when estimated by $C C E M G$ as compared to $C C E P$. Deletion of some of the macro variables from return equation does not change the remaining estimated coefficients significantly, and the results seem to be quite robust. The coefficients of the cross sectional averages of the explanatory variables are generally less significant than the region specific variables. In contrast, the cross section average of the dependant variable is highly significant and its coefficient is very close to one. Its inclusion is clearly critical in dealing with the unobserved common factors and can be viewed as proxing for the market index as in Capital Asset Pricing Models (CAPM).

\section{Conclusions}

Much of the empirical research carried out on panels assume some form of cross section error independence. However, such assumptions are usually suspect, in practice, and as a result recent advances in the theoretical literature have focused on the analysis of cross sectional dependence.

In this paper we explore further some aspects of the work by Pesaran (2004) who has develop methods for estimation and inference in panel data models with multifactor error structures. The method is based on proxying unobserved factors with cross sectional averages. We compare this method with alternative methods that aim to augment panel regressions with factor estimates using principal components. We reach two major conclusions. Firstly, methods based on principal components do not seem to work as well as the methods based on cross sectional averages. The estimation error for the factor estimates seems to be one, but not the only, reason for this inferior performance. Using Monte Carlo experiments we show that the PC augmented estimators could still be subject to substantial small sample bias due to the need to selecting a sub-set of factors for inclusion in the model to be estimated. 
The relevance of CCE type estimators is illustrated by an empirical application to a rich panel of company returns with a wide geographical coverage. The CCE approach allows us to estimate asset return equations with observed as well as unobserved common factors; thus going beyond CAPM and asset pricing models that focus exclusively on observed or unobserved factors. The empirical results clearly show the importance of country-specific macro variables for the analysis of company returns beyond the market indices.

\section{References}

Anselin, L. (2001): "Spatial Econometrics," in A Companion to Theoretical Econometrics, ed. by B. Baltagi. Blackwell, Oxford.

BAI, J. (2003): "Inferential Theory for Factor Models of Large Dinensions," Econometrica, $71,135-173$.

BAI, J., AND S. NG (2002): "Determining the Number of Factors in Approximate Factor Models," Econometrica, 70, 191-221.

Chamberlain, G., and M. Rothschild (1983): "Arbitrage, Factor Structure and MeanVariance Analysis in Large Asset Markets," Econometrica, 51.

Coakley, J., A. Fuertes, and R. P. Smith (2002): "A Principal Components Approach to Cross-Section Dependence in Panels," Mimeo, Birkbeck College, University of London.

_ (2004): "Unobserved Heterogeneity in Panel Time Series Models," Forthcoming in Computational Statistics and Data Analysis.

Conley, T. G., And B. Dupor (2003): "A Spatial Analysis of Sectoral Complementarity," Journal of Political Economy, 111, 311-352.

Conley, T. G., And G. Topa (2002): "Socio-economic Distance and Spatial Patterns in Unemployment," Journal of Applied Econometrics, 17, 303-327.

Conor, G., And R. Korajzcyk (1986): "Performance Measurement with the Arbitrage Pricing Theory: A New Framework for Analysis," Journal of Financial Economics, 15, 373-394.

(1988): "Risk and Return in an Equilibrium APT: Application to a new test methodology," Journal of Financial Economics, 21, 255-289. 
Forni, M., M. Hallin, M. Lippi, And L. Reichlin (2000): "The Generalised Factor Model: Identification and Estimation," Review of Economics and Statistics, 82, 540-554.

Forni, M., And L. Reichlin (1998): "Let's Get Real: A Factor Analytical Approach to Disaggregated Business Cycle Dynamics," Review of Economic Studies, 65, 453-473.

Lee, K. C., And M. H. Pesaran (1993): "The Role of Sectoral Interactions in Wage Determination in the UK Economy," The Economic Journal, 103, 21-55.

Pesaran, M. H. (2004): "Estimation and Inference in Large Heterogeneous panels with a Multifactor Error Structure," University of Cambridge, Available at http://www.econ.cam.ac.uk/faculty/pesaran/CDlargePesaran.pdf.

Pesaran, M. H., T. Schuermann, and B. J. Treutler (2004): "The Role of Industry, Geography and Firm Heterogeneity in Credit Risk Diversification," Paper presented at NBER Conference on Risks in Financial Institutions.

Pesaran, M. H., T. Schuermann, and S. M. Weiner (2004): "Modeling Regional Interdependencies using a Global Error-Correcting Macroeconomic Model," Journal of Business Economics and Statistics, (with Discussions and a Rejoinder), 22, 129-181.

Pesaran, M. H., and R. P. Smith (1995): "Estimating Long-Run Relationships from Dynamic Heterogeneous Panels," Journal of Econometrics, 68, 79-113.

Stock, J. H., and M. W. Watson (2002): "Macroeconomic Forecasting Using Diffusion Indices," Journal of Business and Economic Statistics, 20, 147-162.

Stone, R. (1947): "On the Interdependence of Blocks of Transactions," Supplement of the Journal of the Royal Statistical Society, 9, 1-45. 
Table 1. Naive Estimator

\begin{tabular}{|c|c|c|c|c|c|c|c|c|c|c|}
\hline & & & \multicolumn{4}{|c|}{ One Factor } & \multicolumn{4}{|c|}{ Two Factors } \\
\hline \multicolumn{2}{|c|}{$\# x$} & $N / T$ & 30 & 50 & 100 & 200 & 30 & 50 & 100 & 200 \\
\hline & \multirow{4}{*}{1} & 30 & 2731.1 & 2535.7 & 2648.3 & 2781.2 & 5516.9 & 5250.6 & 5163.0 & 5124.6 \\
\hline B & & 50 & 2675.2 & 2722.7 & 2874.8 & 2660.2 & 5270.2 & 5084.6 & 5187.1 & 5387.0 \\
\hline $\mathrm{i}$ & & 100 & 2729.7 & 2732.6 & 2688.7 & 2660.6 & 4874.1 & 5491.7 & 5363.7 & 5904.4 \\
\hline a & & 200 & 2814.9 & 2738.9 & 2602.4 & 2743.4 & 5941.5 & 4977.3 & 5749.6 & 5615.7 \\
\hline \multirow[t]{4}{*}{$\mathrm{s}$} & \multirow{4}{*}{3} & 30 & 1195.2 & 1133.3 & 1198.2 & 1160.4 & 2261.5 & 2302.0 & 2100.7 & 2032.9 \\
\hline & & 50 & 1210.6 & 1190.5 & 1146.1 & 1153.1 & 2056.7 & 2100.8 & 2152.4 & 2076.8 \\
\hline & & 100 & 1154.9 & 1280.3 & 1191.2 & 1200.7 & 2046.2 & 2097.5 & 2139.9 & 2089.8 \\
\hline & & 200 & 1244.8 & 1160.3 & 1153.9 & 1158.4 & 2019.4 & 2063.2 & 2113.6 & 2161.1 \\
\hline \multirow{4}{*}{$\begin{array}{c}\mathrm{R} \\
\mathrm{M} \\
\mathrm{S}\end{array}$} & \multirow{4}{*}{1} & 30 & 29.2 & 26.5 & 27.0 & 28.1 & 56.3 & 53.2 & 52.0 & 51.4 \\
\hline & & 50 & 28.2 & 28.2 & 29.2 & 26.8 & 53.7 & 51.5 & 52.2 & 54.0 \\
\hline & & 100 & 28.5 & 28.1 & 27.3 & 26.8 & 49.8 & 55.4 & 53.9 & 59.2 \\
\hline & & 200 & 29.3 & 28.1 & 26.4 & 27.6 & 60.1 & 50.3 & 57.7 & 56.3 \\
\hline \multirow[t]{4}{*}{$\mathrm{E}$} & \multirow{4}{*}{3} & 30 & 19.6 & 17.5 & 14.4 & 12.9 & 32.7 & 28.8 & 23.7 & 21.6 \\
\hline & & 50 & 17.9 & 14.7 & 13.1 & 12.5 & 26.9 & 24.2 & 23.2 & 21.6 \\
\hline & & 100 & 14.7 & 14.8 & 12.8 & 12.4 & 23.6 & 22.8 & 22.2 & 21.4 \\
\hline & & 200 & 14.2 & 12.6 & 12.1 & 11.8 & 22.0 & 21.6 & 21.6 & 21.8 \\
\hline \multirow{4}{*}{$\begin{array}{l}\mathrm{S} \\
\mathrm{i}\end{array}$} & \multirow{4}{*}{1} & 30 & 91.0 & 97.7 & 100.0 & 100.0 & 100.0 & 100.0 & 100.0 & 100.0 \\
\hline & & 50 & 97.1 & 99.2 & 100.0 & 100.0 & 100.0 & 100.0 & 100.0 & 100.0 \\
\hline & & 100 & 99.2 & 100.0 & 100.0 & 100.0 & 100.0 & 100.0 & 100.0 & 100.0 \\
\hline & & 200 & 99.8 & 100.0 & 100.0 & 100.0 & 100.0 & 100.0 & 100.0 & 100.0 \\
\hline \multirow[t]{4}{*}{ e } & \multirow{4}{*}{3} & 30 & 70.7 & 76.2 & 99.4 & 100.0 & 95.3 & 99.9 & 100.0 & 100.0 \\
\hline & & 50 & 79.0 & 96.8 & 100.0 & 100.0 & 97.8 & 100.0 & 100.0 & 100.0 \\
\hline & & 100 & 92.5 & 99.2 & 100.0 & 100.0 & 99.7 & 100.0 & 100.0 & 100.0 \\
\hline & & 200 & 98.8 & 99.9 & 100.0 & 100.0 & 100.0 & 100.0 & 100.0 & 100.0 \\
\hline \multirow{4}{*}{$\begin{array}{c}\mathrm{O} \\
\mathrm{W}\end{array}$} & \multirow{4}{*}{1} & 30 & 82.0 & 92.1 & 100.0 & 100.0 & 100.0 & 100.0 & 100.0 & 100.0 \\
\hline & & 50 & 92.7 & 97.2 & 100.0 & 100.0 & 100.0 & 100.0 & 100.0 & 100.0 \\
\hline & & 100 & 97.0 & 99.5 & 100.0 & 100.0 & 99.9 & 100.0 & 100.0 & 100.0 \\
\hline & & 200 & 98.0 & 99.9 & 100.0 & 100.0 & 100.0 & 100.0 & 100.0 & 100.0 \\
\hline e & & 30 & 40.4 & 38.8 & 77.0 & 93.8 & 84.3 & 98.2 & 99.9 & 100.0 \\
\hline \multirow[t]{3}{*}{$\mathrm{r}$} & 3 & 50 & 48.7 & 71.1 & 84.0 & 96.7 & 90.0 & 99.2 & 100.0 & 100.0 \\
\hline & & 100 & 65.2 & 83.9 & 96.9 & 99.9 & 97.9 & 100.0 & 100.0 & 100.0 \\
\hline & & 200 & 84.1 & 91.8 & 98.6 & 100.0 & 99.3 & 100.0 & 100.0 & 100.0 \\
\hline
\end{tabular}


Table 1 (continued). Naive Estimator

\begin{tabular}{|c|c|c|c|c|c|c|c|c|c|c|}
\hline & & & \multicolumn{4}{|c|}{ Three Factors } & \multicolumn{4}{|c|}{ Four Factors } \\
\hline \multicolumn{2}{|c|}{$\# x$} & $N / T$ & 30 & 50 & 100 & 200 & 30 & 50 & 100 & 200 \\
\hline & \multirow{4}{*}{1} & 30 & 7030.9 & 7007.8 & 6742.8 & 7187.6 & 7776.8 & 7975.1 & 7721.5 & 7839.8 \\
\hline B & & 50 & 6919.5 & 7141.9 & 6585.5 & 7151.4 & 8143.6 & 7462.9 & 7740.1 & 7879.7 \\
\hline $\mathrm{i}$ & & 100 & 6247.5 & 6858.1 & 6785.6 & 6704.2 & 7515.4 & 7433.3 & 8082.8 & 7913.0 \\
\hline $\mathrm{a}$ & & 200 & 7034.4 & 6590.4 & 7326.2 & 7223.1 & 7983.9 & 8055.9 & 8039.8 & 8373.7 \\
\hline \multirow[t]{4}{*}{$\mathrm{S}$} & \multirow{4}{*}{3} & 30 & 2558.5 & 2532.6 & 2729.7 & 2669.0 & 2818.1 & 2849.3 & 2838.3 & 2758.1 \\
\hline & & 50 & 2568.3 & 2618.9 & 2706.2 & 2640.4 & 2839.9 & 2725.2 & 2854.4 & 2864.6 \\
\hline & & 100 & 2517.6 & 2747.9 & 2525.3 & 2428.1 & 2846.2 & 2874.4 & 2893.2 & 2870.4 \\
\hline & & 200 & 2584.7 & 2680.1 & 2618.7 & 2603.4 & 2822.2 & 2773.3 & 2884.4 & 2886.9 \\
\hline \multirow{4}{*}{$\begin{array}{c}\mathrm{R} \\
\mathrm{M} \\
\mathrm{S}\end{array}$} & \multirow{4}{*}{1} & 30 & 71.0 & 70.6 & 67.7 & 72.0 & 78.4 & 80.1 & 77.4 & 78.5 \\
\hline & & 50 & 69.9 & 71.8 & 66.1 & 71.6 & 81.9 & 75.0 & 77.5 & 78.9 \\
\hline & & 100 & 63.2 & 69.0 & 68.1 & 67.1 & 75.7 & 74.6 & 80.9 & 79.2 \\
\hline & & 200 & 70.9 & 66.3 & 73.4 & 72.3 & 80.2 & 80.7 & 80.5 & 83.8 \\
\hline \multirow[t]{4}{*}{$\mathrm{E}$} & \multirow{4}{*}{3} & 30 & 36.9 & 29.7 & 30.0 & 28.1 & 47.5 & 37.0 & 32.6 & 29.2 \\
\hline & & 50 & 33.0 & 30.0 & 28.9 & 27.4 & 40.3 & 31.9 & 31.9 & 30.2 \\
\hline & & 100 & 28.7 & 29.4 & 26.2 & 24.7 & 33.9 & 31.4 & 30.3 & 29.5 \\
\hline & & 200 & 27.6 & 27.8 & 26.6 & 26.2 & 31.2 & 29.5 & 29.6 & 29.3 \\
\hline \multirow{4}{*}{$\begin{array}{l}\mathrm{S} \\
\mathrm{i} \\
\mathrm{z}\end{array}$} & \multirow{4}{*}{1} & 30 & 100.0 & 100.0 & 100.0 & 100.0 & 100.0 & 100.0 & 100.0 & 100.0 \\
\hline & & 50 & 100.0 & 100.0 & 100.0 & 100.0 & 100.0 & 100.0 & 100.0 & 100.0 \\
\hline & & 100 & 100.0 & 100.0 & 100.0 & 100.0 & 100.0 & 100.0 & 100.0 & 100.0 \\
\hline & & 200 & 100.0 & 100.0 & 100.0 & 100.0 & 100.0 & 100.0 & 100.0 & 100.0 \\
\hline \multirow[t]{4}{*}{$\mathrm{e}$} & \multirow{4}{*}{3} & 30 & 98.9 & 100.0 & 100.0 & 100.0 & 97.8 & 100.0 & 100.0 & 100.0 \\
\hline & & 50 & 99.6 & 100.0 & 100.0 & 100.0 & 99.9 & 100.0 & 100.0 & 100.0 \\
\hline & & 100 & 100.0 & 100.0 & 100.0 & 100.0 & 100.0 & 100.0 & 100.0 & 100.0 \\
\hline & & 200 & 100.0 & 100.0 & 100.0 & 100.0 & 100.0 & 100.0 & 100.0 & 100.0 \\
\hline \multirow{4}{*}{$\begin{array}{l}\mathrm{P} \\
\mathrm{O} \\
\mathrm{W}\end{array}$} & \multirow{4}{*}{1} & 30 & 100.0 & 100.0 & 100.0 & 100.0 & 100.0 & 100.0 & 100.0 & 100.0 \\
\hline & & 50 & 100.0 & 100.0 & 100.0 & 100.0 & 100.0 & 100.0 & 100.0 & 100.0 \\
\hline & & 100 & 100.0 & 100.0 & 100.0 & 100.0 & 100.0 & 100.0 & 100.0 & 100.0 \\
\hline & & 200 & 100.0 & 100.0 & 100.0 & 100.0 & 100.0 & 100.0 & 100.0 & 100.0 \\
\hline $\mathrm{e}$ & \multirow{4}{*}{3} & 30 & 94.6 & 100.0 & 100.0 & 100.0 & 93.3 & 99.8 & 100.0 & 100.0 \\
\hline \multirow[t]{3}{*}{$\mathrm{r}$} & & 50 & 98.5 & 100.0 & 100.0 & 100.0 & 98.9 & 100.0 & 100.0 & 100.0 \\
\hline & & 100 & 99.7 & 100.0 & 100.0 & 100.0 & 100.0 & 100.0 & 100.0 & 100.0 \\
\hline & & 200 & 100.0 & 100.0 & 100.0 & 100.0 & 100.0 & 100.0 & 100.0 & 100.0 \\
\hline
\end{tabular}

Notes: The naive estimator is given by $\boldsymbol{\beta}=\left(\sum_{i=1}^{N} \mathbf{X}_{i}^{\prime} \mathbf{M} \mathbf{X}_{i}\right)^{-1} \sum_{i=1}^{N} \mathbf{X}_{i}^{\prime} \mathbf{M} \mathbf{y}_{i}$ where $\mathbf{M}=$ $\mathbf{I}_{T}-\mathbf{D}\left(\mathbf{D}^{\prime} \mathbf{D}\right)^{-1} \mathbf{D}^{\prime}$. Bias and RMSE estimates are scaled up by 10000 and 100, respectively. 
Table 2. Mean Group Estimator

\begin{tabular}{|c|c|c|c|c|c|c|c|c|c|c|}
\hline & & & \multicolumn{4}{|c|}{ One Factor } & \multicolumn{4}{|c|}{ Two Factors } \\
\hline \multicolumn{2}{|c|}{$\# x$} & $N / T$ & 30 & 50 & 100 & 200 & 30 & 50 & 100 & 200 \\
\hline & \multirow{4}{*}{1} & 30 & -1.1 & 202.4 & 155.8 & 155.5 & 160.8 & -64.2 & -24.7 & -62.0 \\
\hline B & & 50 & -5.6 & 112.1 & 115.0 & 19.2 & -6.6 & 70.6 & 30.7 & 8.3 \\
\hline $\mathrm{i}$ & & 100 & 27.0 & 15.9 & 14.3 & 38.7 & 45.9 & -19.3 & 25.9 & -31.0 \\
\hline $\mathrm{a}$ & & 200 & -31.6 & 10.6 & -24.6 & 10.0 & 41.4 & 10.5 & 17.5 & -5.9 \\
\hline \multirow[t]{4}{*}{$\mathrm{s}$} & \multirow{4}{*}{3} & 30 & 87.1 & 117.0 & -106.3 & 36.7 & 166.8 & 160.0 & -52.2 & -184.8 \\
\hline & & 50 & 72.4 & -48.1 & -5.9 & -0.6 & -117.9 & -21.2 & 74.8 & 138.3 \\
\hline & & 100 & 20.6 & -3.3 & -9.4 & 27.0 & -68.1 & -7.1 & 42.4 & -95.9 \\
\hline & & 200 & -57.4 & -32.9 & -8.1 & -0.1 & 4.9 & 0.0 & 2.4 & 26.2 \\
\hline \multirow{4}{*}{$\begin{array}{c}\mathrm{R} \\
\mathrm{M} \\
\mathrm{S}\end{array}$} & \multirow{4}{*}{1} & 30 & 13.7 & 8.8 & 4.8 & 4.4 & 13.3 & 9.9 & 6.9 & 3.9 \\
\hline & & 50 & 9.0 & 7.1 & 5.0 & 2.9 & 11.4 & 8.2 & 4.9 & 3.5 \\
\hline & & 100 & 6.4 & 5.0 & 3.0 & 2.0 & 8.3 & 5.4 & 3.7 & 2.3 \\
\hline & & 200 & 4.7 & 3.3 & 2.2 & 1.5 & 6.2 & 3.8 & 2.5 & 1.8 \\
\hline \multirow[t]{4}{*}{$\mathrm{E}$} & \multirow{4}{*}{3} & 30 & 23.8 & 18.7 & 10.3 & 6.9 & 40.9 & 25.1 & 14.6 & 9.4 \\
\hline & & 50 & 21.0 & 11.9 & 8.1 & 5.9 & 28.7 & 17.1 & 11.7 & 7.6 \\
\hline & & 100 & 14.1 & 9.8 & 5.7 & 3.7 & 19.3 & 13.0 & 7.4 & 5.6 \\
\hline & & 200 & 10.0 & 6.2 & 4.3 & 2.7 & 14.0 & 9.2 & 5.5 & 3.8 \\
\hline \multirow{4}{*}{$\begin{array}{l}\mathrm{S} \\
\mathrm{i} \\
\mathrm{Z}\end{array}$} & \multirow{4}{*}{1} & 30 & 6.8 & 6.0 & 6.2 & 7.3 & 6.6 & 6.6 & 6.2 & 6.7 \\
\hline & & 50 & 6.3 & 5.7 & 6.2 & 5.0 & 6.1 & 6.2 & 6.4 & 5.1 \\
\hline & & 100 & 5.6 & 5.8 & 5.2 & 5.9 & 5.6 & 5.3 & 6.0 & 5.0 \\
\hline & & 200 & 5.1 & 4.8 & 5.8 & 5.5 & 5.5 & 5.7 & 5.5 & 5.2 \\
\hline \multirow[t]{4}{*}{ e } & \multirow{4}{*}{3} & 30 & 9.3 & 8.5 & 10.1 & 9.4 & 7.5 & 10.5 & 10.4 & 9.5 \\
\hline & & 50 & 7.8 & 7.9 & 7.2 & 6.9 & 7.4 & 6.3 & 6.6 & 7.3 \\
\hline & & 100 & 7.0 & 5.5 & 6.1 & 6.3 & 5.5 & 7.0 & 5.7 & 5.3 \\
\hline & & 200 & 5.1 & 5.8 & 5.1 & 4.5 & 5.9 & 5.1 & 6.0 & 5.5 \\
\hline \multirow{4}{*}{$\begin{array}{c}\mathrm{O} \\
\mathrm{W}\end{array}$} & \multirow{4}{*}{1} & 30 & 8.1 & 8.1 & 14.7 & 16.9 & 6.9 & 11.1 & 15.3 & 35.4 \\
\hline & & 50 & 11.1 & 9.4 & 15.4 & 38.9 & 8.5 & 9.3 & 18.2 & 30.1 \\
\hline & & 100 & 12.5 & 18.5 & 36.5 & 65.1 & 9.6 & 16.1 & 26.4 & 62.3 \\
\hline & & 200 & 20.0 & 31.9 & 69.0 & 91.3 & 12.6 & 26.5 & 50.8 & 81.7 \\
\hline e & & 30 & 10.2 & 10.0 & 19.0 & 23.2 & 8.2 & 11.1 & 14.6 & 25.2 \\
\hline \multirow[t]{3}{*}{$\mathrm{r}$} & 3 & 50 & 8.3 & 12.8 & 17.5 & 25.7 & 8.2 & 8.8 & 9.8 & 13.8 \\
\hline & & 100 & 9.8 & 10.8 & 27.6 & 48.1 & 7.4 & 10.0 & 13.5 & 34.4 \\
\hline & & 200 & 11.8 & 22.1 & 38.9 & 78.2 & 8.8 & 12.2 & 25.7 & 42.6 \\
\hline
\end{tabular}


Table 2 (continued). Mean Group Estimator

\begin{tabular}{|c|c|c|c|c|c|c|c|c|c|c|}
\hline & & & \multicolumn{4}{|c|}{ Three Factors } & \multicolumn{4}{|c|}{ Four Factors } \\
\hline \multicolumn{2}{|c|}{$\# x$} & $N / T$ & 30 & 50 & 100 & 200 & 30 & 50 & 100 & 200 \\
\hline & \multirow{4}{*}{1} & 30 & -186.2 & -216.5 & 78.7 & -8.1 & 42.6 & -122.5 & 92.9 & 304.8 \\
\hline B & & 50 & -35.5 & 148.4 & 81.2 & -287.1 & 36.5 & -66.2 & 191.8 & 99.5 \\
\hline $\mathrm{i}$ & & 100 & -78.0 & 75.3 & -80.6 & 35.4 & -56.5 & -137.1 & -7.9 & 21.5 \\
\hline $\mathrm{a}$ & & 200 & -19.1 & -17.9 & 20.3 & -10.0 & -48.6 & -29.7 & 23.5 & 21.8 \\
\hline \multirow[t]{4}{*}{$\mathrm{S}$} & \multirow{4}{*}{3} & 30 & -100.5 & 47.4 & -238.8 & 4.8 & -87.9 & 157.1 & 132.8 & -88.6 \\
\hline & & 50 & 407.0 & -17.0 & -60.7 & -19.4 & -158.6 & 72.5 & -171.6 & 185.7 \\
\hline & & 100 & 187.5 & -17.5 & 0.6 & 46.0 & 23.4 & -89.5 & -118.0 & 24.5 \\
\hline & & 200 & 11.3 & -57.9 & -25.9 & -1.0 & -113.5 & -0.4 & 81.2 & -72.6 \\
\hline & \multirow{4}{*}{1} & 30 & 19.1 & 14.4 & 8.1 & 6.6 & 22.8 & 14.5 & 10.0 & 8.2 \\
\hline $\mathrm{R}$ & & 50 & 15.3 & 9.5 & 7.6 & 6.0 & 16.6 & 13.0 & 8.0 & 5.6 \\
\hline M & & 100 & 10.0 & 7.3 & 4.9 & 3.6 & 12.5 & 8.7 & 6.5 & 3.7 \\
\hline $\mathrm{S}$ & & 200 & 7.4 & 5.1 & 3.7 & 2.5 & 9.0 & 6.3 & 3.9 & 2.8 \\
\hline \multirow[t]{4}{*}{$\mathrm{E}$} & \multirow{4}{*}{3} & 30 & 44.7 & 23.5 & 17.5 & 11.8 & 63.8 & 35.6 & 22.6 & 13.2 \\
\hline & & 50 & 36.1 & 22.4 & 13.9 & 9.9 & 48.5 & 26.0 & 20.5 & 13.4 \\
\hline & & 100 & 23.7 & 16.0 & 9.3 & 5.8 & 32.9 & 19.6 & 13.2 & 9.3 \\
\hline & & 200 & 15.9 & 11.8 & 6.6 & 4.2 & 23.0 & 15.6 & 9.4 & 6.5 \\
\hline \multirow{4}{*}{$\begin{array}{l}\mathrm{S} \\
\mathrm{i} \\
\mathrm{z}\end{array}$} & \multirow{4}{*}{1} & 30 & 5.9 & 6.4 & 6.8 & 5.4 & 6.5 & 5.2 & 5.5 & 6.6 \\
\hline & & 50 & 5.9 & 5.4 & 5.7 & 7.2 & 5.3 & 4.7 & 7.4 & 5.0 \\
\hline & & 100 & 5.1 & 5.2 & 6.2 & 5.5 & 5.1 & 5.4 & 5.1 & 5.2 \\
\hline & & 200 & 5.1 & 5.1 & 5.1 & 5.1 & 5.2 & 5.5 & 4.7 & 5.5 \\
\hline \multirow[t]{4}{*}{ e } & \multirow{4}{*}{3} & 30 & 10.7 & 8.7 & 10.8 & 9.7 & 8.5 & 8.3 & 9.3 & 9.7 \\
\hline & & 50 & 8.5 & 7.8 & 7.5 & 9.5 & 7.5 & 8.2 & 7.9 & 9.2 \\
\hline & & 100 & 5.3 & 5.9 & 4.7 & 6.6 & 5.6 & 5.4 & 6.1 & 5.2 \\
\hline & & 200 & 4.9 & 5.3 & 5.5 & 5.8 & 5.6 & 6.3 & 5.3 & 5.5 \\
\hline \multirow{4}{*}{$\begin{array}{c}\mathrm{P} \\
\mathrm{O} \\
\mathrm{W}\end{array}$} & \multirow{4}{*}{1} & 30 & 7.8 & 9.5 & 10.7 & 12.3 & 7.0 & 8.2 & 8.1 & 6.6 \\
\hline & & 50 & 8.0 & 7.6 & 10.5 & 30.5 & 6.3 & 7.5 & 8.8 & 10.4 \\
\hline & & 100 & 8.9 & 9.2 & 23.9 & 28.8 & 8.2 & 11.5 & 13.1 & 25.7 \\
\hline & & 200 & 10.2 & 18.2 & 27.0 & 54.2 & 10.3 & 14.6 & 22.9 & 43.0 \\
\hline $\mathrm{e}$ & & 30 & 11.1 & 10.0 & 14.7 & 16.1 & 8.6 & 9.0 & 9.9 & 15.2 \\
\hline \multirow[t]{3}{*}{$\mathrm{r}$} & 3 & 50 & 8.3 & 8.6 & 11.1 & 16.2 & 8.1 & 8.7 & 9.8 & 10.8 \\
\hline & & 100 & 5.5 & 7.5 & 11.2 & 20.2 & 6.2 & 7.6 & 11.1 & 11.6 \\
\hline & & 200 & 7.1 & 9.9 & 19.8 & 38.9 & 7.5 & 7.6 & 9.5 & 21.9 \\
\hline
\end{tabular}

Notes: The Mean Group estimator is given by (14). Its estimated variance is given by (18). Bias and RMSE estimates are scaled up by 10000 and 100, respectively. 
Table 3. Pooled Estimator

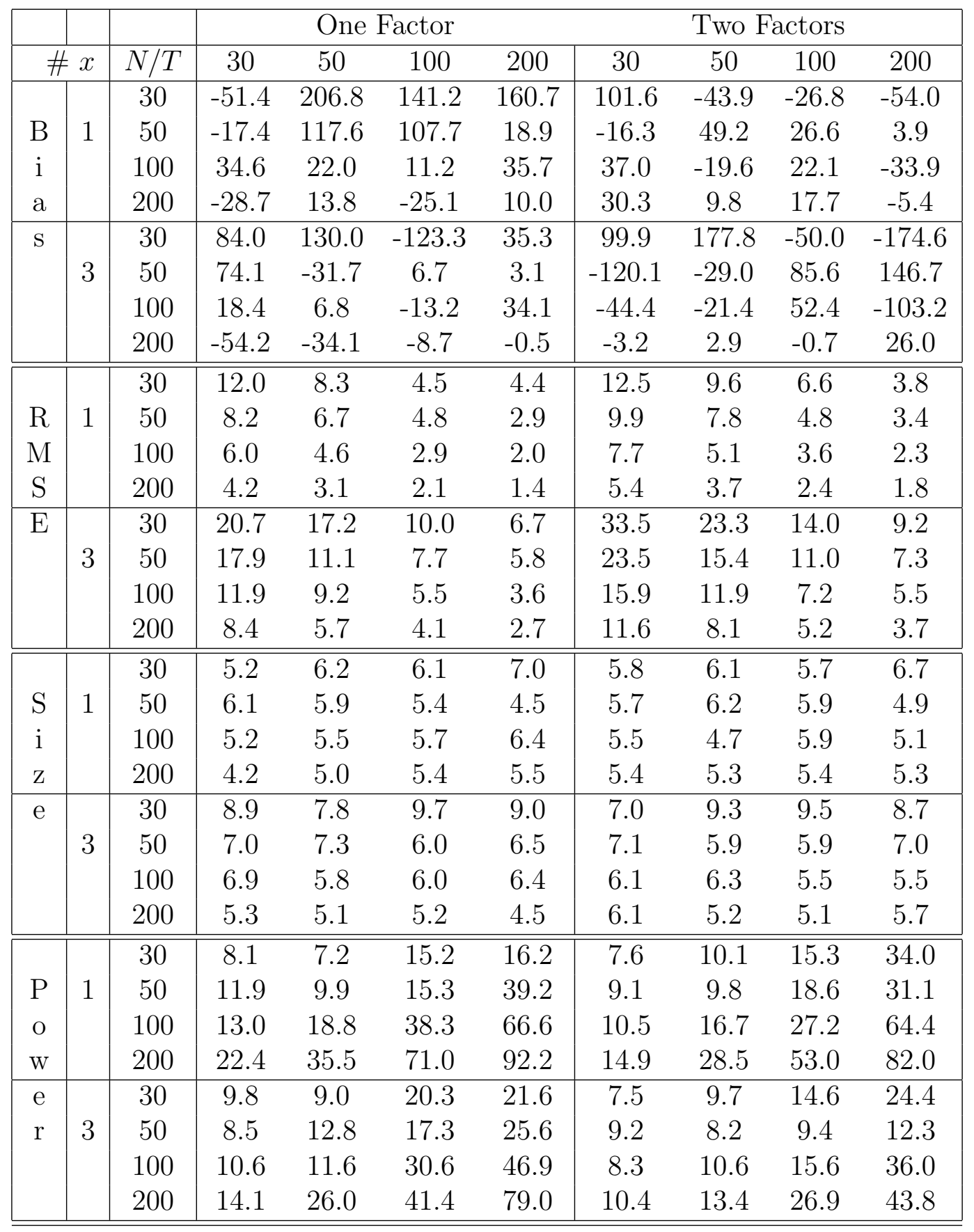


Table 3 (continued). Pooled Estimator

\begin{tabular}{|c|c|c|c|c|c|c|c|c|c|c|}
\hline & & & \multicolumn{4}{|c|}{ Three Factors } & \multicolumn{4}{|c|}{ Four Factors } \\
\hline \multicolumn{2}{|c|}{$\# x$} & $N / T$ & 30 & 50 & 100 & 200 & 30 & 50 & 100 & 200 \\
\hline & \multirow{4}{*}{1} & 30 & -206.9 & -277.0 & 53.3 & -5.3 & 13.1 & -121.2 & 93.8 & 314.9 \\
\hline B & & 50 & -3.9 & 139.5 & 77.2 & -288.9 & 115.5 & -140.6 & 203.0 & 110.4 \\
\hline $\mathrm{i}$ & & 100 & -81.1 & 45.7 & -75.4 & 32.6 & -53.4 & -131.1 & 0.2 & 19.9 \\
\hline $\mathrm{a}$ & & 200 & -10.0 & -15.0 & 17.7 & -10.3 & -36.6 & -33.9 & 25.0 & 20.7 \\
\hline \multirow[t]{4}{*}{$\mathrm{S}$} & \multirow{4}{*}{3} & 30 & -101.6 & 55.2 & -270.4 & 0.4 & -28.2 & 160.3 & 146.7 & -177.8 \\
\hline & & 50 & 382.2 & -18.3 & -71.6 & -23.7 & -179.5 & 45.0 & -219.9 & 220.3 \\
\hline & & 100 & 146.4 & -23.8 & -9.1 & 39.7 & 27.3 & -86.4 & -144.0 & 33.9 \\
\hline & & 200 & 20.7 & -37.7 & -21.7 & 0.5 & -116.9 & 11.4 & 83.2 & -76.4 \\
\hline \multirow{4}{*}{$\begin{array}{c}\mathrm{R} \\
\mathrm{M} \\
\mathrm{S}\end{array}$} & \multirow{4}{*}{1} & 30 & $\overline{17.4}$ & 13.5 & 7.8 & 6.6 & 22.2 & 13.8 & 9.8 & 8.0 \\
\hline & & 50 & 13.6 & 9.1 & 7.4 & 5.9 & 15.8 & 12.6 & 7.7 & 5.6 \\
\hline & & 100 & 9.3 & 6.9 & 4.9 & 3.5 & 11.6 & 8.1 & 6.3 & 3.6 \\
\hline & & 200 & 6.8 & 4.9 & 3.5 & 2.4 & 8.2 & 5.9 & 3.8 & 2.8 \\
\hline \multirow[t]{4}{*}{$\mathrm{E}$} & \multirow{4}{*}{3} & 30 & 38.1 & 21.2 & 17.0 & 11.6 & 55.0 & 32.3 & 21.3 & 13.0 \\
\hline & & 50 & 30.9 & 19.9 & 13.2 & 9.9 & 41.7 & 23.6 & 19.4 & 13.4 \\
\hline & & 100 & 19.4 & 14.5 & 8.9 & 5.6 & 27.5 & 17.9 & 12.6 & 9.0 \\
\hline & & 200 & 13.4 & 10.5 & 6.3 & 4.1 & 19.6 & 14.0 & 9.0 & 6.4 \\
\hline \multirow{4}{*}{$\begin{array}{l}\mathrm{S} \\
\mathrm{i} \\
\mathrm{z}\end{array}$} & \multirow{4}{*}{1} & 30 & 6.5 & 5.9 & 6.2 & 5.5 & 6.0 & 4.7 & 4.8 & 6.0 \\
\hline & & 50 & 5.5 & 5.5 & 5.3 & 7.1 & 5.3 & 3.8 & 7.4 & 4.7 \\
\hline & & 100 & 4.9 & 4.9 & 5.9 & 5.3 & 5.1 & 5.1 & 5.0 & 5.1 \\
\hline & & 200 & 4.3 & 5.1 & 5.0 & 5.7 & 5.0 & 5.0 & 4.2 & 5.2 \\
\hline \multirow[t]{4}{*}{$\mathrm{e}$} & \multirow{4}{*}{3} & 30 & 9.2 & 8.5 & 9.8 & 8.3 & 7.6 & 7.6 & 8.1 & 8.3 \\
\hline & & 50 & 7.5 & 6.9 & 7.4 & 8.8 & 7.3 & 7.3 & 7.1 & 9.0 \\
\hline & & 100 & 6.2 & 4.9 & 4.5 & 5.5 & 6.6 & 5.3 & 6.8 & 5.7 \\
\hline & & 200 & 5.1 & 4.9 & 5.3 & 5.6 & 5.5 & 5.8 & 5.5 & 5.1 \\
\hline \multirow{4}{*}{$\begin{array}{c}\mathrm{P} \\
\mathrm{O} \\
\mathrm{W}\end{array}$} & \multirow{4}{*}{1} & 30 & 7.5 & 9.6 & 10.5 & 12.7 & 6.4 & 7.6 & 6.9 & 5.9 \\
\hline & & 50 & 6.9 & 7.5 & 10.6 & 29.8 & 6.3 & 7.7 & 9.2 & 9.8 \\
\hline & & 100 & 9.3 & 9.7 & 24.1 & 29.4 & 8.8 & 12.2 & 13.0 & 26.0 \\
\hline & & 200 & 10.7 & 19.2 & 28.1 & 55.5 & 11.2 & 15.2 & 23.8 & 43.5 \\
\hline $\mathrm{e}$ & & 30 & 10.2 & 9.0 & 15.8 & 15.5 & 8.6 & 7.2 & 8.8 & 14.5 \\
\hline \multirow[t]{3}{*}{$\mathrm{r}$} & 3 & 50 & 7.4 & 7.5 & 11.4 & 16.1 & 7.3 & 8.2 & 10.0 & 10.7 \\
\hline & & 100 & 6.8 & 8.3 & 11.3 & 21.0 & 6.8 & 7.8 & 12.2 & 11.7 \\
\hline & & 200 & 7.4 & 10.8 & 20.3 & 40.6 & 7.5 & 8.6 & 9.4 & 22.6 \\
\hline
\end{tabular}

Notes: The Pooled estimator is given by (19). Its estimated variance is given by (20). Bias and RMSE estimates are scaled up by 10000 and 100, respectively. 
Table 4. MGPC Estimator

\begin{tabular}{|c|c|c|c|c|c|c|c|c|c|c|}
\hline & & & \multicolumn{5}{|c|}{ One Factor } & \multicolumn{2}{|c|}{ Two Factors } & \\
\hline \multicolumn{2}{|c|}{$\# x$} & $N / T$ & 30 & 50 & 100 & 200 & 30 & 50 & 100 & 200 \\
\hline & \multirow{4}{*}{1} & 30 & -1877.3 & -1506.8 & -864.4 & -838.1 & -2738.5 & -2469.0 & -2309.2 & -1708.0 \\
\hline B & & 50 & -965.3 & -920.6 & -710.7 & -458.7 & -1960.2 & -1710.8 & -1307.8 & -1085.9 \\
\hline $\mathrm{i}$ & & 100 & -519.3 & -440.1 & -250.8 & -190.9 & -1111.3 & -950.8 & -709.3 & -543.9 \\
\hline a & & 200 & -334.9 & -240.0 & -134.7 & -99.9 & -683.0 & -441.2 & -334.1 & -274.7 \\
\hline \multirow[t]{4}{*}{$\mathrm{S}$} & \multirow{4}{*}{3} & 30 & -1316.3 & -1172.1 & -925.7 & -840.8 & -3103.8 & -2581.6 & -2327.2 & -2055.3 \\
\hline & & 50 & -977.6 & -629.1 & -563.0 & -569.7 & -1794.1 & -1346.7 & -1257.1 & -1141.8 \\
\hline & & 100 & -454.1 & -382.7 & -264.0 & -223.4 & -858.0 & -730.2 & -567.4 & -507.4 \\
\hline & & 200 & -284.2 & -186.9 & -141.5 & -117.5 & -440.4 & -378.7 & -267.0 & -245.8 \\
\hline \multirow{4}{*}{$\begin{array}{c}\mathrm{R} \\
\mathrm{M} \\
\mathrm{S}\end{array}$} & \multirow{4}{*}{1} & 30 & 23.8 & 17.6 & 10.0 & 9.5 & 30.9 & 26.7 & 24.2 & 17.6 \\
\hline & & 50 & 13.8 & 11.9 & 8.9 & 5.5 & 23.3 & 19.3 & 14.0 & 11.4 \\
\hline & & 100 & 8.7 & 6.9 & 4.0 & 2.8 & 14.4 & 11.3 & 8.1 & 5.9 \\
\hline & & 200 & 6.2 & 4.3 & 2.6 & 1.8 & 10.0 & 6.1 & 4.2 & 3.3 \\
\hline \multirow[t]{4}{*}{$\mathrm{E}$} & \multirow{4}{*}{3} & 30 & 28.8 & 22.9 & 14.1 & 11.0 & 54.0 & 36.5 & 28.0 & 22.9 \\
\hline & & 50 & 24.2 & 13.9 & 10.1 & 8.3 & 36.7 & 23.2 & 17.9 & 14.0 \\
\hline & & 100 & 15.2 & 10.7 & 6.4 & 4.3 & 23.4 & 16.0 & 9.7 & 7.7 \\
\hline & & 200 & 10.9 & 6.7 & 4.6 & 3.0 & 16.3 & 10.7 & 6.3 & 4.7 \\
\hline \multirow{4}{*}{$\begin{array}{l}\text { S } \\
\mathrm{i} \\
\mathrm{z}\end{array}$} & \multirow{4}{*}{1} & 30 & 28.2 & 38.9 & 40.2 & 50.7 & 51.5 & 66.1 & 87.4 & 91.6 \\
\hline & & 50 & 19.8 & 24.4 & 30.6 & 33.9 & 37.0 & 47.7 & 71.9 & 81.0 \\
\hline & & 100 & 12.9 & 15.3 & 12.3 & 16.4 & 23.8 & 36.4 & 44.4 & 60.2 \\
\hline & & 200 & 10.3 & 10.3 & 9.5 & 10.9 & 16.9 & 19.4 & 26.0 & 32.8 \\
\hline \multirow[t]{4}{*}{ e } & \multirow{4}{*}{3} & 30 & 16.6 & 16.1 & 27.7 & 41.4 & 21.8 & 33.8 & 58.5 & 75.8 \\
\hline & & 50 & 11.5 & 13.7 & 17.0 & 26.6 & 14.3 & 19.9 & 30.8 & 51.8 \\
\hline & & 100 & 8.1 & 7.4 & 10.2 & 13.2 & 8.9 & 13.2 & 16.0 & 23.4 \\
\hline & & 200 & 6.7 & 7.8 & 6.6 & 8.2 & 7.4 & 7.3 & 9.0 & 14.1 \\
\hline \multirow{4}{*}{$\begin{array}{c}\mathrm{O} \\
\mathrm{W}\end{array}$} & \multirow{4}{*}{1} & 30 & 39.6 & 62.3 & 77.5 & 85.7 & 65.3 & 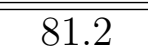 & 97.2 & 999.5 \\
\hline & & 50 & 36.9 & 48.5 & 66.0 & 88.8 & 50.7 & 69.5 & 94.2 & 99.2 \\
\hline & & 100 & 34.1 & 45.9 & 67.5 & 92.7 & 43.9 & 67.1 & 87.9 & 99.5 \\
\hline & & 200 & 36.3 & 56.6 & 82.0 & 97.8 & 39.8 & 61.6 & 91.0 & 98.8 \\
\hline e & & 30 & 23.8 & 27.4 & 55.8 & 79.8 & 26.8 & 44.7 & 77.7 & 93.1 \\
\hline \multirow[t]{3}{*}{$\mathrm{r}$} & 3 & 50 & 17.6 & 29.4 & 46.4 & 74.2 & 19.4 & 32.6 & 53.9 & 84.5 \\
\hline & & 100 & 16.2 & 24.1 & 51.0 & 82.7 & 14.2 & 24.4 & 49.5 & 75.4 \\
\hline & & 200 & 17.2 & 32.0 & 55.9 & 91.7 & 13.9 & 22.6 & 49.7 & 79.5 \\
\hline
\end{tabular}


Table 4 (continued). MGPC Estimator

\begin{tabular}{|c|c|c|c|c|c|c|c|c|c|c|}
\hline & & & \multicolumn{4}{|c|}{ Three Factors } & \multicolumn{4}{|c|}{ Four Factors } \\
\hline \multicolumn{2}{|c|}{$\# x$} & $N / T$ & 30 & 50 & 100 & 200 & 30 & 50 & 100 & 200 \\
\hline & \multirow{4}{*}{1} & 30 & -4314.2 & -3592.8 & -2765.2 & -2715.0 & -4983.8 & -4425.2 & -3710.9 & -3216.8 \\
\hline B & & 50 & -3000.3 & -2351.1 & -2277.3 & -1647.5 & -3506.5 & -3231.9 & -2487.2 & -2103.8 \\
\hline $\mathrm{i}$ & & 100 & -1775.9 & -1529.4 & -1261.9 & -1048.8 & -2490.2 & -1963.1 & -1660.2 & -1268.0 \\
\hline a & & 200 & -1134.3 & -877.5 & -755.9 & -606.6 & -1626.8 & -1311.2 & -976.3 & -733.2 \\
\hline \multirow[t]{4}{*}{$\mathrm{s}$} & \multirow{4}{*}{3} & 30 & -4279.5 & -3433.0 & -3279.3 & -2924.4 & -5472.5 & -4863.3 & -4106.5 & -4141.1 \\
\hline & & 50 & -2690.1 & -2549.2 & -2179.6 & -2031.2 & -4207.6 & -3260.3 & -3375.8 & -2675.5 \\
\hline & & 100 & -1366.1 & -1452.1 & -1064.6 & -704.0 & -2591.5 & -2163.3 & -1927.6 & -1667.4 \\
\hline & & 200 & -757.1 & -692.4 & -479.5 & -405.4 & -1350.0 & -1114.0 & -981.3 & -949.1 \\
\hline \multirow{4}{*}{$\begin{array}{c}\mathrm{R} \\
\mathrm{M} \\
\mathrm{S}\end{array}$} & \multirow{4}{*}{1} & 30 & 47.0 & 38.2 & 28.7 & 27.9 & 53.9 & 46.3 & 38.3 & 32.9 \\
\hline & & 50 & 34.2 & 25.4 & 24.0 & 17.2 & 38.9 & 34.7 & 25.9 & 21.7 \\
\hline & & 100 & 21.1 & 17.3 & 13.6 & 11.1 & 28.6 & 21.5 & 17.7 & 13.2 \\
\hline & & 200 & 14.3 & 10.5 & 8.4 & 6.6 & 19.5 & 14.7 & 10.6 & 7.8 \\
\hline \multirow[t]{4}{*}{$\mathrm{E}$} & \multirow{4}{*}{3} & 30 & 63.0 & 42.8 & 37.1 & 31.3 & 80.4 & 59.1 & 45.7 & 43.5 \\
\hline & & 50 & 47.3 & 35.2 & 26.1 & 22.6 & 66.6 & 42.9 & 39.2 & 29.4 \\
\hline & & 100 & 31.4 & 23.4 & 14.6 & 9.4 & 46.6 & 31.1 & 23.8 & 19.2 \\
\hline & & 200 & 20.5 & 15.1 & 8.5 & 6.0 & 30.9 & 21.5 & 14.2 & 11.7 \\
\hline \multirow{4}{*}{$\begin{array}{l}\mathrm{S} \\
\mathrm{i}\end{array}$} & \multirow{4}{*}{1} & 30 & 66.1 & 78.0 & 91.5 & 97.4 & 71.1 & 89.9 & 96.7 & 99.1 \\
\hline & & 50 & 49.8 & 69.0 & 85.3 & 89.3 & 56.0 & 71.5 & 91.6 & 97.0 \\
\hline & & 100 & 34.8 & 49.6 & 71.9 & 82.8 & 44.6 & 58.6 & 75.5 & 93.7 \\
\hline & & 200 & 24.7 & 34.6 & 51.9 & 68.7 & 35.4 & 51.5 & 68.4 & 75.3 \\
\hline \multirow[t]{4}{*}{$\mathrm{e}$} & \multirow{4}{*}{3} & 30 & 31.4 & 52.0 & 79.3 & 93.0 & 32.0 & 58.2 & 84.3 & 99.0 \\
\hline & & 50 & 18.3 & 33.8 & 56.9 & 85.2 & 24.6 & 38.1 & 69.9 & 87.8 \\
\hline & & 100 & 11.2 & 20.8 & 31.4 & 35.2 & 16.0 & 28.4 & 50.5 & 73.1 \\
\hline & & 200 & 8.2 & 10.5 & 15.7 & 24.8 & 10.8 & 14.2 & 25.9 & 49.5 \\
\hline \multirow{4}{*}{$\begin{array}{c}\mathrm{O} \\
\mathrm{W}\end{array}$} & \multirow{4}{*}{1} & 30 & 73.6 & 86.4 & 97.7 & 99.8 & 78.5 & 94.8 & 99.0 & 99.9 \\
\hline & & 50 & 61.9 & 83.8 & 95.3 & 99.0 & 68.1 & 82.7 & 98.0 & 99.9 \\
\hline & & 100 & 51.2 & 73.1 & 94.2 & 99.2 & 58.4 & 78.6 & 92.7 & 99.9 \\
\hline & & 200 & 45.5 & 67.7 & 91.8 & 99.4 & 53.9 & 78.0 & 95.4 & 99.2 \\
\hline $\mathrm{e}$ & & 30 & 36.9 & 63.8 & 89.8 & 98.7 & 36.5 & 66.0 & 91.6 & 99.9 \\
\hline \multirow[t]{3}{*}{$\mathrm{r}$} & 3 & 50 & 23.2 & 45.1 & 77.5 & 96.8 & 28.8 & 49.2 & 82.0 & 97.2 \\
\hline & & 100 & 16.3 & 33.2 & 61.1 & 82.7 & 21.4 & 40.9 & 71.9 & 93.2 \\
\hline & & 200 & 15.5 & 23.4 & 51.2 & 86.6 & 15.4 & 24.8 & 55.8 & 87.6 \\
\hline
\end{tabular}

Notes: The MGPC estimator is given by (24). Its estimated variance is given by (27). Bias and RMSE estimates are scaled up by 10000 and 100, respectively. 
Table 5. MGCFS Estimator

\begin{tabular}{|c|c|c|c|c|c|c|c|c|c|c|}
\hline & & & \multicolumn{4}{|c|}{ One Factor } & \multicolumn{4}{|c|}{ Two Factors } \\
\hline \multicolumn{2}{|c|}{$\# x$} & $N / T$ & 30 & 50 & 100 & 200 & 30 & 50 & 100 & 200 \\
\hline & \multirow{5}{*}{1} & 30 & 1978.2 & 1538.2 & 1599.3 & 1575.1 & 4630.8 & 3942.8 & 3965.2 & 3836.3 \\
\hline B & & 50 & 1801.9 & 1793.0 & 1730.4 & 1645.1 & 4018.8 & 3795.7 & 3906.6 & 4126.9 \\
\hline $\mathrm{i}$ & & 100 & 1614.9 & 1702.2 & 1619.7 & 1661.7 & 3685.1 & 4248.5 & 3860.1 & 4193.3 \\
\hline $\mathrm{a}$ & & 200 & 1845.4 & 1722.0 & 1516.4 & 1549.1 & 4578.0 & 3639.0 & 3710.2 & 3529.6 \\
\hline s & & 30 & 1007.6 & 991.5 & 1015.0 & 923.7 & 2170.4 & 2193.5 & 1898.8 & 1860.1 \\
\hline & \multirow[t]{4}{*}{3} & 50 & 1092.8 & 1027.7 & 949.0 & 933.3 & 1917.8 & 1983.0 & 1941.0 & 1889.6 \\
\hline & & 100 & 974.8 & 1081.6 & 960.9 & 930.9 & 1873.4 & 1962.2 & 1910.0 & 1830.3 \\
\hline & & 200 & 1034.7 & 944.5 & 919.3 & 950.8 & 1838.3 & 1841.6 & 1885.1 & 1924.1 \\
\hline & & 30 & 23.4 & 17.3 & 16.8 & 16.4 & 47.9 & 40.6 & 40.3 & 38.6 \\
\hline $\mathrm{R}$ & \multirow[t]{4}{*}{1} & 50 & 20.3 & 19.6 & 18.1 & 16.8 & 41.8 & 39.0 & 39.5 & 41.5 \\
\hline M & & 100 & 17.8 & 18.1 & 16.7 & 16.9 & 38.2 & 43.1 & 38.9 & 42.1 \\
\hline S & & 200 & 19.8 & 18.0 & 15.6 & 15.7 & 46.5 & 36.9 & 37.3 & 35.4 \\
\hline $\mathrm{E}$ & & 30 & 21.9 & 19.2 & 13.7 & 11.2 & 38.3 & 30.3 & 23.0 & 20.4 \\
\hline & \multirow[t]{4}{*}{3} & 50 & 19.9 & 14.4 & 12.0 & 10.8 & 29.6 & 24.5 & 22.0 & 20.1 \\
\hline & & 100 & 15.1 & 13.8 & 10.9 & 9.9 & 24.4 & 22.5 & 20.2 & 19.0 \\
\hline & & 200 & 13.3 & 11.0 & 10.0 & 9.9 & 21.5 & 20.1 & 19.5 & 19.6 \\
\hline & & 30 & 70.2 & 76.2 & 97.4 & 98.4 & 99.2 & 99.8 & 100.0 & 100.0 \\
\hline$\overline{S \mathrm{~S}}$ & \multirow[t]{3}{*}{1} & 50 & 81.2 & 87.7 & 98.2 & 100.0 & 99.1 & 100.0 & 100.0 & 100.0 \\
\hline $\mathrm{i}$ & & 100 & 87.5 & 95.7 & 99.9 & 100.0 & 99.7 & 100.0 & 100.0 & 100.0 \\
\hline z & & 200 & 95.9 & 99.5 & 100.0 & 100.0 & 100.0 & 100.0 & 100.0 & 100.0 \\
\hline \multirow[t]{4}{*}{ e } & \multirow{4}{*}{3} & 30 & 54.0 & 62.9 & 89.6 & 96.8 & 88.5 & 98.4 & 99.8 & 100.0 \\
\hline & & 50 & 59.2 & 83.9 & 93.2 & 99.0 & 89.1 & 99.2 & 100.0 & 100.0 \\
\hline & & 100 & 73.7 & 90.8 & 99.6 & 100.0 & 96.5 & 100.0 & 100.0 & 100.0 \\
\hline & & 200 & 90.0 & 96.1 & 99.7 & 100.0 & 99.1 & 100.0 & 100.0 & 100.0 \\
\hline \multirow{4}{*}{$\begin{array}{c}\mathrm{P} \\
\mathrm{O} \\
\mathrm{W}\end{array}$} & \multirow{4}{*}{1} & 30 & 54.9 & 56.1 & 84.5 & 87.5 & 98.2 & 98.9 & 100.0 & 100.0 \\
\hline & & 50 & 65.8 & 73.8 & 88.1 & 98.8 & 97.7 & 99.5 & 100.0 & 100.0 \\
\hline & & 100 & 70.3 & 83.1 & 96.0 & 100.0 & 97.9 & 100.0 & 100.0 & 100.0 \\
\hline & & 200 & 86.6 & 94.6 & 98.3 & 100.0 & 100.0 & 100.0 & 100.0 & 100.0 \\
\hline $\mathrm{e}$ & \multirow{4}{*}{3} & 30 & 33.5 & 36.5 & 55.0 & 62.5 & 76.4 & 92.2 & 97.3 & 99.7 \\
\hline \multirow[t]{3}{*}{$\mathrm{r}$} & & 50 & 33.3 & 49.4 & 55.9 & 66.8 & 73.4 & 95.0 & 99.1 & 100.0 \\
\hline & & 100 & 40.9 & 58.7 & 72.4 & 89.2 & 86.4 & 98.2 & 100.0 & 100.0 \\
\hline & & 200 & 57.5 & 65.6 & 79.5 & 96.2 & 94.7 & 99.6 & 100.0 & 100.0 \\
\hline
\end{tabular}


Table 5 (continued). MGCFS Estimator

\begin{tabular}{|c|c|c|c|c|c|c|c|c|c|c|}
\hline & & & \multicolumn{4}{|c|}{ Three Factors } & \multicolumn{4}{|c|}{ Four Factors } \\
\hline \multicolumn{2}{|c|}{$\# x$} & $N / T$ & 30 & 50 & 100 & 200 & 30 & 50 & 100 & 200 \\
\hline & \multirow{4}{*}{1} & 30 & 6296.5 & 5910.7 & 5636.6 & 6304.7 & 6843.9 & 7350.1 & 6940.5 & 7000.9 \\
\hline B & & 50 & 6136.7 & 5681.3 & 5462.4 & 6054.7 & 7450.9 & 6418.1 & 6900.7 & 6966.7 \\
\hline $\mathrm{i}$ & & 100 & 5084.7 & 5789.9 & 5617.7 & 5334.9 & 6518.3 & 6556.3 & 7084.1 & 6643.9 \\
\hline $\mathrm{a}$ & & 200 & 5681.8 & 5209.1 & 5660.9 & 5080.3 & 6781.6 & 6903.4 & 6454.9 & 5811.4 \\
\hline \multirow[t]{4}{*}{$\mathrm{S}$} & \multirow{4}{*}{3} & 30 & 2478.7 & 2420.8 & 2594.4 & 2492.3 & 2763.9 & 2727.2 & 2713.3 & 2657.8 \\
\hline & & 50 & 2436.1 & 2500.8 & 2563.8 & 2509.8 & 2759.7 & 2648.8 & 2721.6 & 2680.6 \\
\hline & & 100 & 2407.7 & 2591.5 & 2336.2 & 2212.3 & 2759.4 & 2779.2 & 2709.6 & 2744.7 \\
\hline & & 200 & 2429.9 & 2532.0 & 2432.0 & 2418.7 & 2711.9 & 2586.8 & 2731.4 & 2768.9 \\
\hline \multirow{4}{*}{$\begin{array}{c}\mathrm{R} \\
\mathrm{M} \\
\mathrm{S}\end{array}$} & \multirow{4}{*}{1} & 30 & 64.2 & 60.1 & 56.8 & 63.3 & 69.9 & 74.0 & 69.8 & 70.2 \\
\hline & & 50 & 62.5 & 57.4 & 55.1 & 60.7 & 75.2 & 65.0 & 69.2 & 69.8 \\
\hline & & 100 & 51.9 & 58.4 & 56.4 & 53.5 & 66.0 & 66.0 & 71.0 & 66.5 \\
\hline & & 200 & 57.5 & 52.5 & 56.7 & 50.9 & 68.3 & 69.2 & 64.7 & 58.2 \\
\hline \multirow[t]{4}{*}{$\mathrm{E}$} & \multirow{4}{*}{3} & 30 & 42.7 & 30.9 & 29.8 & 27.0 & 57.4 & 40.1 & 33.3 & 29.0 \\
\hline & & 50 & 37.6 & 30.9 & 28.2 & 26.5 & 47.7 & 34.0 & 32.3 & 29.1 \\
\hline & & 100 & 30.5 & 29.2 & 24.8 & 22.7 & 38.6 & 32.3 & 29.3 & 28.6 \\
\hline & & 200 & 27.6 & 27.1 & 25.0 & 24.5 & 32.8 & 29.1 & 28.5 & 28.3 \\
\hline \multirow{4}{*}{$\begin{array}{c}\text { S } \\
\mathrm{i} \\
\mathrm{z}\end{array}$} & \multirow{4}{*}{1} & 30 & 99.8 & 100.0 & 100.0 & 100.0 & 99.7 & 100.0 & 100.0 & 100.0 \\
\hline & & 50 & 100.0 & 100.0 & 100.0 & 100.0 & 100.0 & 100.0 & 100.0 & 100.0 \\
\hline & & 100 & 100.0 & 100.0 & 100.0 & 100.0 & 100.0 & 100.0 & 100.0 & 100.0 \\
\hline & & 200 & 100.0 & 100.0 & 100.0 & 100.0 & 100.0 & 100.0 & 100.0 & 100.0 \\
\hline \multirow[t]{4}{*}{$\mathrm{e}$} & \multirow{4}{*}{3} & 30 & 93.7 & 100.0 & 100.0 & 100.0 & 93.0 & 99.9 & 100.0 & 100.0 \\
\hline & & 50 & 96.8 & 100.0 & 100.0 & 100.0 & 98.2 & 100.0 & 100.0 & 100.0 \\
\hline & & 100 & 99.8 & 100.0 & 100.0 & 100.0 & 99.6 & 100.0 & 100.0 & 100.0 \\
\hline & & 200 & 100.0 & 100.0 & 100.0 & 100.0 & 99.9 & 100.0 & 100.0 & 100.0 \\
\hline \multirow{4}{*}{$\begin{array}{c}\mathrm{P} \\
\mathrm{o} \\
\mathrm{W}\end{array}$} & \multirow{4}{*}{1} & 30 & 99.6 & $\overline{999.9}$ & 100.0 & 100.0 & $\overline{999.4}$ & 100.0 & 100.0 & 100.0 \\
\hline & & 50 & 99.9 & 100.0 & 100.0 & 100.0 & 100.0 & 100.0 & 100.0 & 100.0 \\
\hline & & 100 & 99.7 & 100.0 & 100.0 & 100.0 & 100.0 & 100.0 & 100.0 & 100.0 \\
\hline & & 200 & 100.0 & 100.0 & 100.0 & 100.0 & 100.0 & 100.0 & 100.0 & 100.0 \\
\hline $\mathrm{e}$ & & 30 & 85.4 & 99.1 & 100.0 & 100.0 & 85.9 & 98.7 & 100.0 & 100.0 \\
\hline \multirow[t]{3}{*}{$\mathrm{r}$} & 3 & 50 & 91.6 & 99.7 & 100.0 & 100.0 & 94.9 & 99.7 & 100.0 & 100.0 \\
\hline & & 100 & 97.9 & 100.0 & 100.0 & 100.0 & 98.2 & 100.0 & 100.0 & 100.0 \\
\hline & & 200 & 99.7 & 100.0 & 100.0 & 100.0 & 99.9 & 100.0 & 100.0 & 100.0 \\
\hline
\end{tabular}

Notes: The MGCFS estimator is given by (24), where the factor in (23) has been constructed as described in Section 3.2. Its estimated variance is given by (27). Bias and RMSE estimates are scaled up by 10000 and 100, respectively. 
Table 6. PPC Estimator

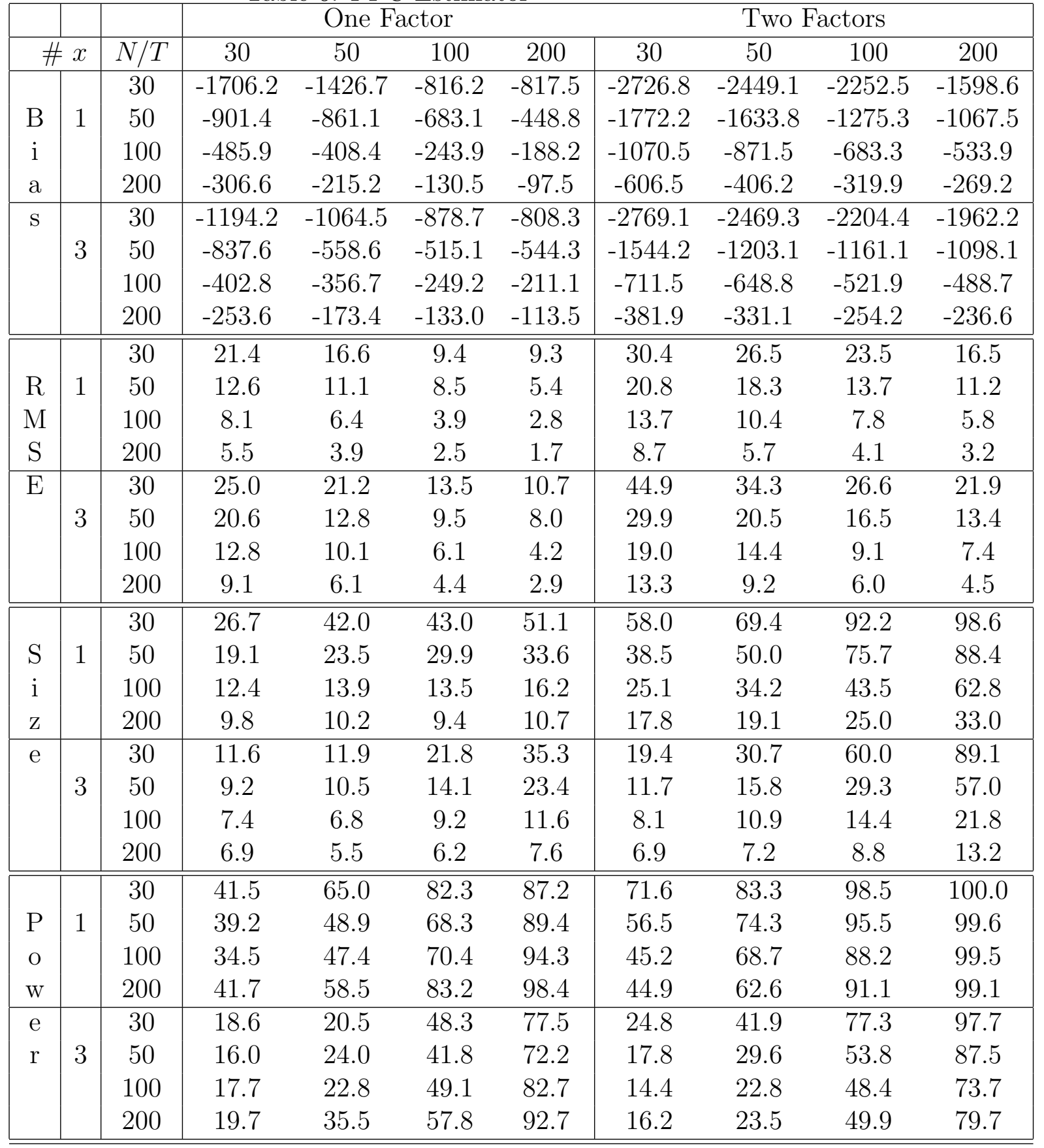


Table 6 (continued). PPC Estimator

\begin{tabular}{|c|c|c|c|c|c|c|c|c|c|c|}
\hline & & & \multicolumn{4}{|c|}{ Three Factors } & \multicolumn{4}{|c|}{ Four Factors } \\
\hline \multicolumn{2}{|c|}{$\# x$} & $N / T$ & 30 & 50 & 100 & 200 & 30 & 50 & 100 & 200 \\
\hline & \multirow{4}{*}{1} & 30 & -4226.6 & -3570.6 & -2714.9 & -2722.1 & -5059.8 & -4391.2 & -3680.7 & -3150.6 \\
\hline B & & 50 & -2882.2 & -2335.7 & -2244.0 & -1645.0 & -3478.5 & -3255.2 & -2423.7 & -2085.1 \\
\hline i & & 100 & -1722.6 & -1477.2 & -1254.3 & -1040.8 & -2426.5 & -1906.8 & -1637.2 & -1258.4 \\
\hline $\mathrm{a}$ & & 200 & -1072.0 & -834.6 & -744.5 & -599.1 & -1526.6 & -1278.4 & -960.8 & -731.9 \\
\hline \multirow[t]{4}{*}{$\mathrm{s}$} & \multirow{4}{*}{3} & 30 & -4073.5 & -3173.2 & -3211.5 & -2903.4 & -5342.3 & -4699.9 & -4029.9 & -4174.5 \\
\hline & & 50 & -2475.2 & -2318.8 & -2095.2 & -1983.0 & -3934.3 & -3149.9 & -3280.7 & -2706.3 \\
\hline & & 100 & -1191.2 & -1285.7 & -1005.0 & -683.5 & -2312.8 & -1970.8 & -1848.8 & -1641.1 \\
\hline & & 200 & -663.1 & -603.2 & -436.9 & -388.1 & -1198.8 & -1008.4 & -925.4 & -921.6 \\
\hline \multirow{4}{*}{$\begin{array}{c}\mathrm{R} \\
\mathrm{M} \\
\mathrm{S}\end{array}$} & \multirow{4}{*}{1} & 30 & 45.6 & 37.8 & 28.2 & 28.0 & 54.3 & 45.8 & 38.0 & 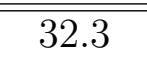 \\
\hline & & 50 & 32.5 & 25.1 & 23.6 & 17.1 & 38.2 & 34.8 & 25.2 & 21.5 \\
\hline & & 100 & 20.2 & 16.6 & 13.5 & 11.0 & 27.5 & 20.8 & 17.5 & 13.1 \\
\hline & & 200 & 13.4 & 10.0 & 8.3 & 6.5 & 18.0 & 14.3 & 10.4 & 7.8 \\
\hline \multirow[t]{4}{*}{$\mathrm{E}$} & \multirow{4}{*}{3} & 30 & 57.7 & 39.2 & 36.2 & 31.0 & 74.2 & 56.1 & 44.6 & 43.7 \\
\hline & & 50 & 41.1 & 31.3 & 25.0 & 22.1 & 59.1 & 40.4 & 37.8 & 29.6 \\
\hline & & 100 & 24.9 & 20.8 & 13.9 & 9.1 & 38.9 & 28.1 & 22.7 & 18.8 \\
\hline & & 200 & 17.1 & 13.1 & 8.0 & 5.8 & 26.1 & 19.1 & 13.4 & 11.4 \\
\hline \multirow{4}{*}{$\begin{array}{l}\mathrm{S} \\
\mathrm{i} \\
\mathrm{z}\end{array}$} & \multirow{4}{*}{1} & 30 & 70.4 & 83.8 & 96.9 & 99.1 & 75.9 & 93.5 & 98.6 & 99.8 \\
\hline & & 50 & 53.3 & 73.4 & 88.2 & 94.0 & 60.2 & 75.3 & 94.7 & 98.2 \\
\hline & & 100 & 39.0 & 52.2 & 72.9 & 85.4 & 49.4 & 63.0 & 77.1 & 95.6 \\
\hline & & 200 & 24.6 & 34.2 & 53.9 & 70.3 & 39.0 & 52.0 & 69.5 & 77.5 \\
\hline \multirow[t]{4}{*}{$\mathrm{e}$} & \multirow{4}{*}{3} & 30 & 29.1 & 53.0 & 84.8 & 98.8 & 29.4 & 63.1 & 89.9 & 100.0 \\
\hline & & 50 & 17.5 & 35.1 & 61.7 & 89.5 & 23.7 & 41.5 & 74.5 & 94.3 \\
\hline & & 100 & 11.4 & 19.7 & 30.9 & 34.2 & 16.1 & 28.0 & 53.0 & 75.4 \\
\hline & & 200 & 8.9 & 10.5 & 13.2 & 22.6 & 10.7 & 13.4 & 24.6 & 50.0 \\
\hline \multirow{4}{*}{$\begin{array}{c}\mathrm{P} \\
\mathrm{O} \\
\mathrm{W}\end{array}$} & \multirow{4}{*}{1} & 30 & 80.3 & 92.0 & 99.4 & 99.9 & 81.9 & 97.7 & 99.6 & 100.0 \\
\hline & & 50 & 66.0 & 86.9 & 97.2 & 99.5 & 71.0 & 86.4 & 99.0 & 99.9 \\
\hline & & 100 & 56.3 & 77.5 & 94.8 & 99.4 & 65.0 & 82.4 & 94.0 & 100.0 \\
\hline & & 200 & 49.4 & 68.7 & 93.2 & 99.2 & 58.2 & 80.3 & 96.0 & 99.5 \\
\hline $\mathrm{e}$ & & 30 & 36.1 & 64.1 & 92.9 & 99.9 & 35.5 & 71.8 & 95.7 & 100.0 \\
\hline \multirow[t]{3}{*}{$\mathrm{r}$} & 3 & 50 & 22.9 & 48.5 & 81.6 & 98.3 & 28.5 & 52.3 & 86.2 & 98.8 \\
\hline & & 100 & 17.6 & 34.6 & 60.7 & 81.8 & 22.6 & 43.2 & 73.7 & 94.2 \\
\hline & & 200 & 15.9 & 23.7 & 50.8 & 86.3 & 17.2 & 25.9 & 55.9 & 87.8 \\
\hline
\end{tabular}

Notes: The PPC estimator is given by (25). Its estimated variance is given by (28). Bias and RMSE estimates are scaled up by 10000 and 100, respectively. 
Table 7. PCFS Estimator

\begin{tabular}{|c|c|c|c|c|c|c|c|c|c|c|}
\hline & & & \multicolumn{4}{|c|}{ One Factor } & \multicolumn{4}{|c|}{ Two Factors } \\
\hline \multicolumn{2}{|c|}{$\# x$} & $N / T$ & 30 & 50 & 100 & 200 & 30 & 50 & 100 & 200 \\
\hline & \multirow{4}{*}{1} & 30 & 1875.6 & 1491.1 & 1574.0 & 1554.9 & 4466.1 & 3822.6 & 3890.2 & 3758.7 \\
\hline B & & 50 & 1728.1 & 1738.4 & 1695.6 & 1625.9 & 3880.7 & 3676.8 & 3815.0 & 4067.3 \\
\hline $\mathrm{i}$ & & 100 & 1538.8 & 1638.8 & 1587.0 & 1632.3 & 3533.3 & 4109.9 & 3780.7 & 4166.3 \\
\hline $\mathrm{a}$ & & 200 & 1755.5 & 1658.1 & 1482.0 & 1526.5 & 4422.2 & 3534.4 & 3672.7 & 3520.8 \\
\hline \multirow[t]{4}{*}{$\mathrm{s}$} & \multirow{4}{*}{3} & 30 & 986.2 & 970.8 & 1015.5 & 921.8 & 2158.9 & 2176.3 & 1900.8 & 1858.4 \\
\hline & & 50 & 1064.6 & 1022.1 & 948.4 & 938.3 & 1889.2 & 1951.5 & 1940.0 & 1887.7 \\
\hline & & 100 & 957.2 & 1071.5 & 961.6 & 935.4 & 1848.2 & 1958.9 & 1912.4 & 1827.2 \\
\hline & & 200 & 1017.0 & 942.2 & 918.8 & 952.6 & 1817.1 & 1831.7 & 1878.5 & 1921.1 \\
\hline \multirow{4}{*}{$\begin{array}{l}\mathrm{R} \\
\mathrm{M} \\
\mathrm{S}\end{array}$} & \multirow{4}{*}{1} & 30 & 22.2 & 16.9 & 16.5 & 16.1 & 46.2 & 39.4 & 39.5 & 37.9 \\
\hline & & 50 & 19.4 & 18.9 & 17.8 & 16.6 & 40.3 & 37.8 & 38.6 & 40.9 \\
\hline & & 100 & 17.0 & 17.5 & 16.4 & 16.5 & 36.7 & 41.7 & 38.1 & 41.8 \\
\hline & & 200 & 18.8 & 17.3 & 15.2 & 15.4 & 44.9 & 35.8 & 36.9 & 35.3 \\
\hline \multirow[t]{4}{*}{$\mathrm{E}$} & \multirow{4}{*}{3} & 30 & 19.6 & 17.9 & 13.3 & 11.0 & 34.2 & 29.0 & 22.6 & 20.2 \\
\hline & & 50 & 18.0 & 13.8 & 11.8 & 10.7 & 27.0 & 23.6 & 21.6 & 20.0 \\
\hline & & 100 & 13.8 & 13.3 & 10.8 & 9.9 & 22.7 & 22.0 & 20.1 & 18.9 \\
\hline & & 200 & 12.5 & 10.8 & 10.0 & 9.9 & 20.6 & 19.6 & 19.4 & 19.5 \\
\hline \multirow{4}{*}{$\begin{array}{l}\text { S } \\
\text { i } \\
z\end{array}$} & \multirow{4}{*}{1} & 30 & 69.0 & 75.2 & 97.8 & 99.5 & 99.4 & 99.9 & 100.0 & 100.0 \\
\hline & & 50 & 82.9 & 89.9 & 98.0 & 100.0 & 99.4 & 100.0 & 100.0 & 100.0 \\
\hline & & 100 & 86.7 & 96.3 & 100.0 & 100.0 & 99.7 & 100.0 & 100.0 & 100.0 \\
\hline & & 200 & 96.2 & 99.5 & 100.0 & 100.0 & 100.0 & 100.0 & 100.0 & 100.0 \\
\hline \multirow[t]{4}{*}{$\mathrm{e}$} & \multirow{4}{*}{3} & 30 & 53.2 & 62.4 & 90.3 & 97.7 & 89.8 & 98.1 & 100.0 & 100.0 \\
\hline & & 50 & 65.0 & 87.4 & 94.2 & 99.5 & 91.5 & 99.3 & 100.0 & 100.0 \\
\hline & & 100 & 77.5 & 92.0 & 99.6 & 100.0 & 98.0 & 100.0 & 100.0 & 100.0 \\
\hline & & 200 & 92.8 & 98.0 & 99.9 & 100.0 & 99.7 & 100.0 & 100.0 & 100.0 \\
\hline \multirow{4}{*}{$\begin{array}{l}\mathrm{P} \\
\mathrm{O} \\
\mathrm{W}\end{array}$} & \multirow{4}{*}{1} & 30 & 54.9 & 53.5 & 85.2 & 91.5 & 98.4 & 98.9 & 100.0 & 100.0 \\
\hline & & 50 & 66.3 & 75.2 & 87.5 & 99.1 & 97.9 & 99.4 & 100.0 & 100.0 \\
\hline & & 100 & 67.8 & 83.5 & 96.5 & 100.0 & 98.2 & 100.0 & 100.0 & 100.0 \\
\hline & & 200 & 87.1 & 94.8 & 98.6 & 100.0 & 100.0 & 100.0 & 100.0 & 100.0 \\
\hline $\mathrm{e}$ & \multirow{4}{*}{3} & 30 & 31.3 & 34.8 & 53.2 & 60.6 & 78.0 & 94.1 & 98.0 & 100.0 \\
\hline \multirow[t]{3}{*}{$\mathrm{r}$} & & 50 & 38.6 & 51.2 & 57.5 & 70.5 & 78.5 & 96.3 & 99.5 & 100.0 \\
\hline & & 100 & 45.5 & 62.5 & 75.6 & 91.8 & 90.0 & 99.1 & 100.0 & 100.0 \\
\hline & & 200 & 63.0 & 70.5 & 82.6 & 97.2 & 96.5 & 99.9 & 100.0 & 100.0 \\
\hline
\end{tabular}


Table 7 (continued). PCFS Estimator

\begin{tabular}{|c|c|c|c|c|c|c|c|c|c|c|}
\hline & & & \multicolumn{4}{|c|}{ Three Factors } & \multicolumn{4}{|c|}{ Four Factors } \\
\hline \multicolumn{2}{|c|}{$\# x$} & $N / T$ & 30 & 50 & 100 & 200 & 30 & 50 & 100 & 200 \\
\hline & \multirow{4}{*}{1} & 30 & 6074.4 & 5790.8 & 5532.9 & 6212.3 & 6657.4 & 7177.3 & 6869.6 & 6912.7 \\
\hline B & & 50 & 5952.6 & 5555.0 & 5348.9 & 5990.5 & 7300.6 & 6272.0 & 6799.5 & 883.0 \\
\hline $\mathrm{i}$ & & 100 & 4905.1 & 5653.3 & 5516.6 & 5262.3 & 6330.6 & 6405.8 & 6974.2 & 6617.6 \\
\hline $\mathrm{a}$ & & 200 & 5485.0 & 5088.9 & 5589.3 & 5090.6 & 6596.4 & 6777.7 & 6404.3 & 5854.1 \\
\hline \multirow[t]{4}{*}{$\mathrm{S}$} & \multirow{4}{*}{3} & 30 & 2477.9 & 2423.6 & 2611.9 & 2493.0 & 2771.2 & 2733.3 & 2764.8 & 2675.2 \\
\hline & & 50 & 2433.4 & 2505.1 & 2553.5 & 2505.4 & 2777.4 & 2664.7 & 2740.7 & 2679.2 \\
\hline & & 100 & 2392.1 & 2589.5 & 2337.2 & 2213.0 & 2764.3 & 2788.5 & 2710.6 & 2738.0 \\
\hline & & 200 & 2417.4 & 2535.9 & 2431.3 & 2417.7 & 2706.2 & 2577.6 & 2730.3 & 2777.1 \\
\hline \multirow{4}{*}{$\begin{array}{l}\mathrm{R} \\
\mathrm{M} \\
\mathrm{S}\end{array}$} & \multirow{4}{*}{1} & 30 & 62.0 & 58.8 & 55.8 & 62.3 & 68.0 & 72.3 & 69.1 & 69.3 \\
\hline & & 50 & 60.6 & 56.2 & 54.0 & 60.1 & 73.7 & 63.5 & 68.2 & 69.0 \\
\hline & & 100 & 50.1 & 57.1 & 55.4 & 52.7 & 64.1 & 64.4 & 69.9 & 66.3 \\
\hline & & 200 & 55.5 & 51.3 & 56.0 & 51.0 & 66.4 & 68.0 & 64.2 & 58.6 \\
\hline \multirow[t]{4}{*}{$\mathrm{E}$} & \multirow{4}{*}{3} & 30 & 39.3 & 29.9 & 29.6 & 26.9 & 52.4 & 38.5 & 32.9 & 28.9 \\
\hline & & 50 & 34.4 & 29.9 & 27.8 & 26.4 & 43.3 & 32.7 & 31.8 & 28.8 \\
\hline & & 100 & 28.5 & 28.5 & 24.6 & 22.7 & 35.1 & 31.4 & 29.0 & 28.5 \\
\hline & & 200 & 26.6 & 26.7 & 24.9 & 24.4 & 31.2 & 28.3 & 28.3 & 28.2 \\
\hline \multirow{4}{*}{$\begin{array}{c}\text { S } \\
\mathrm{i} \\
\mathrm{z}\end{array}$} & \multirow{4}{*}{1} & 30 & 99.9 & 100.0 & 100.0 & 100.0 & 99.9 & 100.0 & 100.0 & 100.0 \\
\hline & & 50 & 100.0 & 100.0 & 100.0 & 100.0 & 100.0 & 100.0 & 100.0 & 100.0 \\
\hline & & 100 & 100.0 & 100.0 & 100.0 & 100.0 & 100.0 & 100.0 & 100.0 & 100.0 \\
\hline & & 200 & 100.0 & 100.0 & 100.0 & 100.0 & 100.0 & 100.0 & 100.0 & 100.0 \\
\hline \multirow[t]{4}{*}{$\mathrm{e}$} & \multirow{4}{*}{3} & 30 & 96.5 & 99.9 & 100.0 & 100.0 & 95.0 & 99.9 & 100.0 & 100.0 \\
\hline & & 50 & 97.2 & 100.0 & 100.0 & 100.0 & 99.1 & 100.0 & 100.0 & 100.0 \\
\hline & & 100 & 99.7 & 100.0 & 100.0 & 100.0 & 99.8 & 100.0 & 100.0 & 100.0 \\
\hline & & 200 & 100.0 & 100.0 & 100.0 & 100.0 & 100.0 & 100.0 & 100.0 & 100.0 \\
\hline \multirow{4}{*}{$\begin{array}{c}\mathrm{P} \\
\mathrm{o} \\
\mathrm{w}\end{array}$} & \multirow{4}{*}{1} & 30 & & & & 100.0 & 99.7 & 100.0 & 100.0 & 100.0 \\
\hline & & 50 & 100.0 & 100.0 & 100.0 & 100.0 & 100.0 & 100.0 & 100.0 & 100.0 \\
\hline & & 100 & 99.7 & 100.0 & 100.0 & 100.0 & 100.0 & 100.0 & 100.0 & 100.0 \\
\hline & & 200 & 100.0 & 100.0 & 100.0 & 100.0 & 100.0 & 100.0 & 100.0 & 100.0 \\
\hline $\mathrm{e}$ & \multirow{4}{*}{3} & 30 & 90.5 & 99.6 & 100.0 & 100.0 & 90.0 & 99.2 & 100.0 & 100.0 \\
\hline \multirow[t]{3}{*}{$\mathrm{r}$} & & 50 & 93.0 & 99.9 & 100.0 & 100.0 & 96.8 & 99.8 & 100.0 & 100.0 \\
\hline & & 100 & 98.3 & 100.0 & 100.0 & 100.0 & 99.2 & 100.0 & 100.0 & 100.0 \\
\hline & & 200 & 100.0 & 100.0 & 100.0 & 100.0 & 99.9 & 100.0 & 100.0 & 100.0 \\
\hline
\end{tabular}

Notes: The PCFS estimator is given by (25), where the factor in (23) has been constructed as described in Section 3.2. Its estimated variance is given by (28). Bias and RMSE estimates are scaled up by 10000 and 100, respectively. 
Table 8. Mean Group (MG) Estimators for Experiments B

\begin{tabular}{|c|c|c|c|c|c|c|c|c|c|c|}
\hline & & & \multicolumn{4}{|c|}{ CCEMG Estimator } & \multicolumn{4}{|c|}{ MG(Theil) Estimator } \\
\hline $\mathrm{No}$ & of $x$ & $N / T$ & 30 & 50 & 100 & 200 & 30 & 50 & 100 & 200 \\
\hline B & \multirow{4}{*}{1} & 30 & 3.4 & -27.8 & 106.1 & -252.0 & 2071.7 & 1796.8 & 1880.7 & -3.5 \\
\hline $\mathrm{i}$ & & 50 & 184.4 & -35.9 & -247.4 & 153.0 & 2862.7 & 1440.6 & 547.2 & 1.1 \\
\hline $\mathrm{a}$ & & 100 & 17.6 & 8.5 & -143.4 & -3.3 & 3203.4 & 2849.7 & 676.3 & 4.3 \\
\hline $\mathrm{s}$ & & 200 & -3.5 & 3.1 & 7.7 & -10.3 & 2877.8 & 2597.8 & 1604.2 & -0.2 \\
\hline$\overline{\mathrm{R}}$ & \multirow{4}{*}{1} & 30 & 24.0 & 18.2 & 13.6 & 7.8 & 24.1 & 20.8 & 21.0 & 5.2 \\
\hline M & & 50 & 20.6 & 13.3 & 9.4 & 6.3 & 30.4 & 18.1 & 11.6 & 4.0 \\
\hline $\mathrm{S}$ & & 100 & 14.2 & 9.8 & 6.4 & 4.2 & 33.0 & 29.7 & 12.0 & 2.7 \\
\hline $\mathrm{E}$ & & 200 & 10.0 & 7.0 & 4.8 & 3.1 & 30.5 & 27.7 & 17.1 & 2.0 \\
\hline$\overline{\mathrm{S}}$ & \multirow{4}{*}{1} & 30 & 6.3 & 5.1 & 6.5 & 7.8 & 51.5 & 54.4 & 71.9 & 5.6 \\
\hline i & & 50 & 5.1 & 5.8 & 4.3 & 6.8 & 84.8 & 53.9 & 33.6 & 7.0 \\
\hline Z & & 100 & 5.1 & 5.3 & 5.9 & 5.8 & 98.8 & 97.5 & 44.1 & 6.2 \\
\hline e & & 200 & 4.7 & 5.6 & 5.7 & 5.2 & 94.0 & 97.9 & 97.1 & 5.7 \\
\hline$\overline{\mathrm{P}}$ & \multirow{4}{*}{1} & 30 & 6.6 & 6.5 & 8.0 & 17.2 & 37.6 & 37.8 & 54.8 & 20.1 \\
\hline o & & 50 & 6.7 & 8.6 & 12.8 & 9.6 & 75.9 & 37.3 & 33.1 & 27.8 \\
\hline $\mathrm{w}$ & & 100 & 6.7 & 8.3 & 18.7 & 24.6 & 96.0 & 94.5 & 54.6 & 48.4 \\
\hline \multirow[t]{2}{*}{ er } & & 200 & 8.2 & 12.0 & 18.4 & 39.2 & 88.5 & 92.5 & 88.6 & 71.3 \\
\hline & & & \multicolumn{4}{|c|}{ MG(AIC) Estimator } & \multicolumn{4}{|c|}{ MGT Estimator } \\
\hline $\mathrm{B}$ & \multirow{4}{*}{1} & 30 & 1802.4 & 1367.2 & 731.8 & -3.6 & 6.3 & -58.3 & -29.0 & -3.8 \\
\hline $\mathrm{i}$ & & 50 & 1210.0 & 547.4 & 9.2 & 0.7 & -34.5 & -26.3 & 5.8 & 1.1 \\
\hline $\mathrm{a}$ & & 100 & 641.5 & 405.8 & -26.0 & 4.3 & 24.5 & -33.2 & -26.0 & 4.3 \\
\hline $\mathrm{s}$ & & 200 & 1369.4 & 105.8 & 71.9 & -0.2 & -17.8 & -1.6 & 2.3 & -0.2 \\
\hline$\overline{\overline{\mathrm{R}}}$ & \multirow{4}{*}{1} & 30 & 22.8 & 18.6 & $\overline{12.2}$ & 5.2 & 16.7 & 111.5 & 8.6 & "5.2 \\
\hline M & & 50 & 20.7 & 10.4 & 6.0 & 4.0 & 13.3 & 8.5 & 5.9 & 4.0 \\
\hline $\mathrm{S}$ & & 100 & 16.4 & 10.5 & 4.3 & 2.7 & 9.2 & 6.1 & 4.3 & 2.7 \\
\hline $\mathrm{E}$ & & 200 & 17.7 & 6.0 & 4.5 & 2.0 & 7.1 & 4.3 & 3.1 & 2.0 \\
\hline$\overline{\bar{S}}$ & \multirow{4}{*}{1} & 30 & 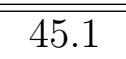 & 41.7 & 25.8 & 5.3 & 5.0 & 5.8 & 5.8 & $\bar{~} 5.7$ \\
\hline $\mathrm{i}$ & & 50 & 36.7 & 17.0 & 5.5 & 6.9 & 5.5 & 5.5 & 5.3 & 7.0 \\
\hline Z & & 100 & 23.6 & 25.9 & 6.3 & 6.2 & 4.5 & 5.3 & 6.3 & 6.2 \\
\hline $\mathrm{e}$ & & 200 & 61.5 & 12.7 & 10.0 & 5.7 & 6.5 & 5.7 & 5.3 & 5.7 \\
\hline$\overline{\overline{\mathrm{P}}}$ & \multirow{4}{*}{1} & $\begin{array}{l}30 \\
\end{array}$ & 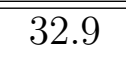 & 29.5 & $\bar{~} 15.0$ & $\bar{~} 19.8$ & "6.5 & 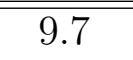 & $\bar{~} 11.3$ & $\overline{20.1}$ \\
\hline o & & 50 & 31.6 & 9.9 & 14.5 & 27.8 & 7.8 & 11.8 & 14.2 & 27.8 \\
\hline $\mathrm{w}$ & & 100 & 26.0 & 25.2 & 25.7 & 48.4 & 8.6 & 15.0 & 25.7 & 48.4 \\
\hline er & & 200 & 46.7 & 27.4 & 40.9 & 71.3 & 12.2 & 22.8 & 37.3 & 71.3 \\
\hline
\end{tabular}

Notes: The $C C E M G$ estimator is given by (14). Its estimated variance is given by (18). The MG(Theil) estimator is given by (24). Its estimated variance is given by (27). The factors included in (23) are chosen by the criterion in (32). The MG(AIC) estimator is given by (24). Its estimated variance is given by (27). The factors included in (23) are chosen by the criterion in (31). The MGT estimator is the MG estimator that uses the true unobserved factor and is therefore, infeasible in practice. 
Table 9. Pooled Estimators for Experiments B

\begin{tabular}{|c|c|c|c|c|c|c|c|c|c|c|}
\hline & & & \multicolumn{4}{|c|}{ CCEP Estimator } & \multicolumn{4}{|c|}{ P(Theil) Estimator } \\
\hline No & of $x$ & $N / T$ & 30 & 50 & 100 & 200 & 30 & 50 & 100 & 200 \\
\hline B & \multirow{4}{*}{1} & 30 & 106.4 & -18.1 & 138.3 & -265.1 & 1994.7 & 1745.6 & 1839.0 & -6.8 \\
\hline $\mathrm{i}$ & & 50 & 237.4 & -23.7 & -240.1 & 156.9 & 2781.7 & 1375.7 & 530.4 & 0.1 \\
\hline $\mathrm{a}$ & & 100 & 20.6 & -4.0 & -133.4 & -2.9 & 3090.8 & 2757.4 & 660.1 & 3.2 \\
\hline $\mathrm{S}$ & & 200 & 2.8 & -7.6 & 7.7 & -11.0 & 2754.0 & 2510.5 & 1564.4 & -1.4 \\
\hline $\mathrm{R}$ & \multirow{4}{*}{1} & 30 & 22.9 & 17.1 & 13.1 & 7.7 & 23.3 & 20.2 & 20.5 & 4.7 \\
\hline M & & 50 & 20.0 & 12.5 & 9.5 & 6.2 & 29.6 & 17.4 & 11.2 & 4.0 \\
\hline $\mathrm{S}$ & & 100 & 13.0 & 9.5 & 6.2 & 4.1 & 31.8 & 28.8 & 11.7 & 2.6 \\
\hline $\mathrm{E}$ & & 200 & 9.0 & 6.5 & 4.5 & 3.0 & 29.2 & 26.8 & 16.7 & 1.9 \\
\hline $\mathrm{S}$ & \multirow{4}{*}{1} & 30 & 5.6 & 5.7 & 6.2 & 7.1 & 47.0 & 53.8 & 71.5 & 4.8 \\
\hline $\mathrm{i}$ & & 50 & 5.5 & 5.3 & 4.8 & 6.2 & 83.5 & 51.2 & 33.1 & 5.8 \\
\hline $\mathrm{Z}$ & & 100 & 4.9 & 5.3 & 5.5 & 5.2 & 98.5 & 97.5 & 44.0 & 5.3 \\
\hline e & & 200 & 5.1 & 6.0 & 5.5 & 5.1 & 94.5 & 97.8 & 97.2 & 5.0 \\
\hline $\mathrm{P}$ & \multirow{4}{*}{1} & 30 & 6.2 & 6.6 & 7.1 & 17.5 & 32.9 & 35.6 & 53.0 & 18.2 \\
\hline $\mathrm{O}$ & & 50 & 6.0 & 8.1 & 11.5 & 9.3 & 73.6 & 35.2 & 33.0 & 24.6 \\
\hline $\mathrm{w}$ & & 100 & 6.9 & 9.3 & 18.9 & 25.1 & 96.0 & 94.2 & 56.4 & 50.0 \\
\hline er & & 200 & 8.5 & 13.5 & 19.6 & 39.7 & 88.4 & 91.8 & 88.3 & 74.8 \\
\hline & & & \multicolumn{4}{|c|}{$\mathrm{P}(\mathrm{AIC})$ Estimator } & \multicolumn{4}{|c|}{ PT Estimator } \\
\hline B & \multirow{4}{*}{1} & 30 & 1734.6 & 1325.4 & 716.0 & -6.6 & -8.9 & -52.8 & -22.6 & -7.2 \\
\hline $\mathrm{i}$ & & 50 & 1165.1 & 514.3 & 6.0 & -0.3 & -44.0 & -34.3 & 2.6 & 0.1 \\
\hline a & & 100 & 609.3 & 388.1 & -24.3 & 3.2 & 16.6 & -37.9 & -24.3 & 3.2 \\
\hline $\mathrm{s}$ & & 200 & 1301.2 & 106.9 & 70.8 & -1.4 & -18.1 & 3.6 & 3.3 & -1.4 \\
\hline $\mathrm{R}$ & \multirow{4}{*}{1} & 30 & 22.0 & 18.0 & 11.9 & 4.7 & 16.0 & 10.7 & 8.3 & 4.7 \\
\hline M & & 50 & 20.1 & 9.9 & 5.6 & 4.0 & 13.2 & 8.1 & 5.5 & 4.0 \\
\hline S & & 100 & 15.7 & 10.2 & 3.9 & 2.6 & 8.5 & 5.9 & 3.9 & 2.6 \\
\hline $\mathrm{E}$ & & 200 & 16.8 & 5.7 & 4.4 & 1.9 & 6.5 & 4.0 & 3.0 & 1.9 \\
\hline S & \multirow{4}{*}{1} & 30 & 39.8 & 40.8 & 22.1 & 4.5 & 4.4 & 4.7 & 4.7 & 4.8 \\
\hline $\mathrm{i}$ & & 50 & 35.4 & 14.6 & 4.8 & 5.7 & 4.6 & 5.2 & 4.6 & 5.8 \\
\hline Z & & 100 & 23.5 & 25.1 & 5.7 & 5.3 & 4.3 & 4.7 & 5.7 & 5.3 \\
\hline e & & 200 & 62.0 & 12.1 & 10.0 & 5.0 & 5.7 & 4.8 & 5.2 & 5.0 \\
\hline$\overline{\mathrm{P}}$ & \multirow{4}{*}{1} & 30 & 28.4 & 27.4 & 12.6 & 17.9 & 5.1 & 7.2 & 9.6 & 18.2 \\
\hline $\mathrm{O}$ & & 50 & 29.1 & 9.0 & 14.3 & 24.6 & 6.9 & 11.2 & 14.1 & 24.6 \\
\hline $\mathrm{w}$ & & 100 & 25.6 & 24.4 & 27.7 & 50.0 & 8.6 & 14.5 & 27.7 & 50.0 \\
\hline er & & 200 & 47.6 & 28.5 & 42.2 & 74.8 & 13.6 & 24.2 & 38.9 & 74.8 \\
\hline
\end{tabular}

Notes: The CCEP estimator is given by (19). Its estimated variance is given by (20). The $\mathrm{P}$ (Theil) estimator is given by (25). Its estimated variance is given by (28). The factors included in (23) are chosen by the criterion in (32). The $\mathrm{P}(\mathrm{AIC})$ estimator is given by (25). Its estimated variance is given by (28). The factors included in (23) are chosen by the criterion in (31). The PT estimator is the pooled estimator that uses the true unobserved factor and is therefore, infeasible in practice. 
Table 10. Empirical Application: Pooled Estimator

\begin{tabular}{|c|c|c|c|c|c|c|c|}
\hline \multicolumn{8}{|c|}{ Table 10. Empirical Application: Pooled Estimator } \\
\hline Spec. & $\Delta q$ & $\Delta \pi$ & $\Delta r$ & $\Delta m$ & $\Delta e$ & const & $\Delta \pi_{o}$ \\
\hline 1 & $1.251_{(4.45)}$ & $0.335_{(1.63)}$ & $-1.594(-1.94)$ & $0.185_{(2.21)}$ & $-0.366_{(-5.12)}$ & $-0.002_{(-0.45)}$ & $0.042_{(1.07)}$ \\
\hline 2 & - & $0.277_{(1.35)}$ & $-1.763(-2.02)$ & $0.283_{(3.30)}$ & $-0.411_{(-5.75)}$ & $0.003_{(0.83)}$ & $0.073_{(1.76)}$ \\
\hline 3 & $1.254_{(4.43)}$ & - & $-1.464(-1.77)$ & $0.164_{(2.03)}$ & $-0.356_{(-4.95)}$ & $-0.003(-0.67)$ & $0.039_{(0.98)}$ \\
\hline 4 & $1.243_{(4.30)}$ & $0.187_{(0.67)}$ & - & $0.167_{(1.95)}$ & $-0.395(-5.51)$ & $-0.002_{(-0.50)}$ & $0.040_{(0.98)}$ \\
\hline 5 & $1.261_{(4.51)}$ & $0.265_{(1.35)}$ & $-1.678_{(-1.89)}$ & - & $-0.336(-4.79)$ & $-0.004_{(-1.00)}$ & $0.039_{(1.01)}$ \\
\hline 6 & $1.669_{(5.67)}$ & $0.087_{(0.41)}$ & $-1.923(-2.43)$ & $0.115_{(1.30)}$ & - & $-0.003_{(-0.70)}$ & $0.032_{(0.80)}$ \\
\hline 7 & $1.252_{(4.49)}$ & - & $-1.583(-1.80)$ & - & $-0.329_{(-4.64)}$ & $-0.004_{(-0.99)}$ & $0.038_{(0.98)}$ \\
\hline
\end{tabular}

\begin{tabular}{|c|c|c|c|c|c|c|c|}
\hline Spec. & rèt & $\overline{\Delta q}$ & $\overline{\Delta \pi}$ & $\overline{\Delta r}$ & $\Delta m$ & $\bar{\Delta} e$ & $\bar{\sigma}_{i}^{2}$ \\
\hline 1 & $1.021_{(26.14)}$ & $-0.482_{(-0.90)}$ & $-0.654_{(-1.68)}$ & $2.022_{(3.35)}$ & $-0.377_{(-1.43)}$ & $0.360_{(4.45)}$ & $0.0328_{(0.0440)}$ \\
\hline 2 & $1.040_{(26.21)}$ & - & $-0.593(-1.61)$ & $2.132_{(3.48)}$ & $-0.552_{(-2.08)}$ & $0.362_{(4.35)}$ & $0.0334_{(0.0443)}$ \\
\hline 3 & $1.016_{(25.67)}$ & $-0.489_{(-0.95)}$ & - & $1.689_{(3.70)}$ & $-0.240(-0.91)$ & $0.324_{(4.08)}$ & $0.0327_{(0.0429)}$ \\
\hline 4 & $1.011_{(25.29)}$ & $-0.598(-1.18)$ & $-0.167_{(-0.76)}$ & - & $-0.267_{(-0.99)}$ & $0.376_{(4.71)}$ & $0.0326_{(0.0421)}$ \\
\hline 5 & $1.018_{(27.82)}$ & $-0.446(-0.83)$ & $-0.515(-1.35)$ & $2.070_{(3.33)}$ & - & $0.319_{(3.96)}$ & $0.0327_{(0.0440)}$ \\
\hline 6 & $1.005_{(28.47)}$ & $-0.564(-1.04)$ & $-0.433(-1.18)$ & $2.415_{(3.57)}$ & $-0.337(-1.29)$ & - & $0.0335_{(0.0439)}$ \\
\hline 7 & $1.015_{(27.43)}$ & $-0.440(-0.85)$ & - & $1.808_{(3.72)}$ & - & $0.295_{(3.71)}$ & $0.0327_{(0.0432)}$ \\
\hline
\end{tabular}

Table 12. Empirical Application: Mean Group Estimator

\begin{tabular}{|c|ccccccc|}
\multicolumn{7}{|c|}{ Table 12. Empirical Application: Mean Group Estimator } \\
\hline & & & & & & \\
Spec. & $\Delta q$ & $\Delta \pi$ & $\Delta r$ & $\Delta m$ & $\Delta e$ & const & $\Delta \pi_{o}$ \\
1 & $1.163_{(3.68)}$ & $0.303_{(0.98)}$ & $-3.041_{(-5.62)}$ & $0.139_{(0.96)}$ & $-0.268_{(-4.02)}$ & $0.001_{(0.19)}$ & $0.055_{(1.48)}$ \\
2 & - & $0.308_{(1.04)}$ & $-3.634_{(-6.62)}$ & $0.194_{(1.54)}$ & $-0.354_{(-6.03)}$ & $0.007_{(1.76)}$ & $0.116_{(2.54)}$ \\
3 & $1.048_{(3.40)}$ & - & $-2.789_{(-4.89)}$ & $0.119_{(0.89)}$ & $-0.284_{(-4.70)}$ & $-0.000_{(-0.06)}$ & $0.043_{(1.18)}$ \\
4 & $1.053_{(3.39)}$ & $0.041_{(0.14)}$ & - & $0.042_{(0.27)}$ & $-0.348_{(-4.84)}$ & $-0.000_{(-0.07)}$ & $0.040_{(1.07)}$ \\
5 & $1.366_{(4.33)}$ & $0.388_{(1.32)}$ & $-3.490_{(-6.35)}$ & - & $-0.198_{(-3.10)}$ & $-0.005_{(-0.94)}$ & $0.015_{(0.38)}$ \\
6 & $1.347_{(4.81)}$ & $0.100_{(0.36)}$ & $-2.917_{(-5.37)}$ & $0.091_{(0.64)}$ & - & $-0.000_{(-0.08)}$ & $0.055_{(1.44)}$ \\
7 & $1.253_{(4.20)}$ & - & $-3.174_{(-5.56)}$ & - & $-0.188_{(-3.23)}$ & $-0.004_{(-0.90)}$ & $0.015_{(0.40)}$ \\
\hline
\end{tabular}

Table 13. Empirical Application: Mean Group Estimator (cont.)

\begin{tabular}{|c|c|c|c|c|c|c|c|}
\hline Spec. & rēet & $\overline{\Delta q}$ & $\Delta \pi$ & $\overline{\Delta r}$ & $\Delta m$ & $\overline{\Delta e}$ & $\chi_{k}^{2}$ \\
\hline 1 & $1.018_{(24.23)}$ & $-0.668(-1.21)$ & $-0.700_{(-1.36)}$ & $-0.148(-0.24)$ & $-0.285(-1.01)$ & $0.170_{(2.23)}$ & 0.00 \\
\hline 2 & $1.058_{(24.97)}$ & - & $-0.965(-1.99)$ & $0.178_{(0.29)}$ & $-0.610(-2.15)$ & $0.170_{(2.27)}$ & 0.00 \\
\hline 3 & $1.013_{(23.32)}$ & $-0.595(-1.05)$ & - & $-0.272_{(-0.43)}$ & $-0.131_{(-0.49)}$ & $0.146_{(2.00)}$ & 0.00 \\
\hline 4 & $1.001_{(23.30)}$ & $-0.440(-0.80)$ & $-0.655(-1.53)$ & - & $-0.064(-0.23)$ & $0.187_{(2.51)}$ & 0.00 \\
\hline 5 & $0.995_{(26.19)}$ & $-0.826_{(-1.60)}$ & $-0.680(-1.44)$ & $-0.243(-0.40)$ & - & $0.143_{(1.89)}$ & 0.00 \\
\hline 6 & $0.993_{(27.57)}$ & $-0.542_{(-1.00)}$ & $-0.162_{(-0.34)}$ & $-0.484(-0.76)$ & $-0.159_{(-0.61)}$ & - & 0.00 \\
\hline 7 & $0.991_{(25.37)}$ & $-0.691_{(-1.29)}$ & - & $-0.390(-0.69)$ & - & $0.136_{(1.91)}$ & 0.00 \\
\hline
\end{tabular}

